Draft Version November 17, 2018

Preprint typeset using $\mathrm{L}_{\mathrm{A}}^{\mathrm{T}} \mathrm{E}$ X style emulateapj v. 11/10/09

\title{
He II $\lambda 4686$ IN $\eta$ CARINAE: COLLAPSE OF THE WIND-WIND COLLISION REGION DURING PERIASTRON PASSAGE
}

M. Teodoro and A. Damineli

Instituto de Astronomia, Geofísica e Ciências Atmosféricas, Universidade de São Paulo, Rua do Matão 1226, Cidade Universitária, São Paulo, 05508-900, Brazil

\section{J. I. ARIAS}

Departamento de Física, Universidad de La Serena, Av. Cisternas 1200 Norte, La Serena, Chile

F. X. DE Araújo

Observatório Nacional, Rua General José Cristino 77, São Cristóvão, Rio de Janeiro, 20921-400, Brazil

R. H. BARBÁ ${ }^{2}$

Instituto de Ciencias Astronómicas, de la Tierra, y del Espacio (ICATE-CONICET), Av. España Sur 1512, J5402DSP San Juan, Argentina

\section{F. CORCORAN ${ }^{3}$}

CRESST and X-ray Astrophysics Laboratory, NASA/Goddard Space Flight Center, Greenbelt, MD 20771, USA

\section{Borges FERnANDES}

Observatório Nacional, Rua General José Cristino 77, São Cristóvão, Rio de Janeiro, 20921-400, Brazil

\section{E. FERnÁNDEZ-LAJÚs ${ }^{4}$}

Facultad de Ciencias Astronómicas y Geofísicas, Universidad Nacional de La Plata, Paseo del Bosque s/n, La Plata, BA, B1900FWA, Argentina

\section{Fraga}

Southern Observatory for Astrophysical Research, Colina El Pino s/n, Casilla 603, La Serena, Chile

R. C. GAMEN ${ }^{4}$

Facultad de Ciencias Astronómicas y Geofísicas, Universidad Nacional de La Plata, Paseo del Bosque s/n, La Plata, BA, B1900FWA, Argentina

\section{J. F. GONZÁLEZ}

Instituto de Ciencias Astronómicas de la Tierra y del Espacio (ICATE-CONICET), Avenida España 1512 Sur, J5402DSP, San Juan, 
J. H. GROH

Max-Planck-Institute für Radioastronomie, Auf dem Hügel 69, D-53121 Bonn, Germany

J. L. MARShall

Department of Physics and Astronomy, Texas A\&M University, College Station, TX 77843-4242, USA

\title{
P. J. MCGREgor
}

Research School of Astronomy and Astrophysics (RSAA), Mount Stromlo Observatory, Cotter Road, Weston, ACT 2611, Australia

\section{N. MoRrell}

Las Campanas Observatory, Observatories of the Carnegie Institution of Washington, Casilla 601, La Serena, Chile

D. C. NiCHOLLS

Research School of Astronomy and Astrophysics (RSAA), Mount Stromlo Observatory, Cotter Road, Weston, ACT 2611, Australia

\section{E. R. PARKIN}

Research School of Astronomy and Astrophysics (RSAA), Mount Stromlo Observatory, Cotter Road, Weston, ACT 2611, Australia

\author{
C. B. Pereira
}

Observatório Nacional, Rua General José Cristino 77, São Cristóvão, Rio de Janeiro, 20921-400, Brazil

\section{M. Phillips}

Las Campanas Observatory, Observatories of the Carnegie Institution of Washington, Casilla 601, La Serena, Chile G. R. Solivella ${ }^{4}$
Facultad de Ciencias Astronómicas y Geofísicas, Universidad Nacional de La Plata, Paseo del Bosque s/n, La Plata, BA, B1900FWA,
Argentina

\section{J. E. STEINER}

Instituto de Astronomia, Geofísica e Ciências Atmosféricas, Universidade de São Paulo, Rua do Matão 1226, Cidade Universitária, São Paulo, 05508-900, Brazil

\section{STRITZINGER ${ }^{5}$}

The Oskar Klein Centre, Department of Astronomy, Stockholm University, AlbaNova, 10691 Stockholm, Sweden

I. THOMPSON

Observatories of the Carnegie Institution, 813 Santa Barbara Street, Pasadena, California 91101, USA

C. A. O. Torres

Laboratório Nacional de Astrofísica, Rua Estados Unidos 154, Bairro das Nações, 37504-364, Itajubá, Brazil

M. A. P. TORres ${ }^{6}$

Harvard-Smithsonian Center for Astrophysics, 60 Garden Street, Cambridge, MA 02138, USA

M. I. Zevallos Herencia

Observatório Nacional, Rua General José Cristino 77, São Cristóvão, Rio de Janeiro, 20921-400, Brazil

Draft version November 17, 2018

\section{ABSTRACT}

The periodic spectroscopic events in $\eta$ Carinae are now well established and occur near the periastron passage of two massive stars in a very eccentric orbit. Several mechanisms have been proposed to explain the variations of different spectral features, such as an eclipse by the wind-wind collision boundary, a shell ejection from the primary star or accretion of its wind onto the secondary. All 
of them have problems explaining all the observed phenomena. To better understand the nature of the cyclic events, we performed a dense monitoring of $\eta$ Carinae with 5 Southern telescopes during the 2009 low excitation event, resulting in a set of data of unprecedented quality and sampling. The intrinsic luminosity of the He II $\lambda 4686$ emission line $\left(L \sim 310 L_{\odot}\right)$ just before periastron reveals the presence of a very luminous transient source of extreme UV radiation emitted in the wind-wind collision (WWC) region. Clumps in the primary's wind probably explain the flare-like behavior of both the X-ray and He II $\lambda 4686$ light-curves. After a short-lived minimum, He II $\lambda 4686$ emission rises again to a new maximum, when X-rays are still absent or very weak. We interpret this as a collapse of the WWC onto the "surface" of the secondary star, switching off the hard X-ray source and diminishing the WWC shock cone. The recovery from this state is controlled by the momentum balance between the secondary's wind and the clumps in the primary's wind.

Subject headings: line: profiles - stars: early-type - stars: individual ( $\eta$ Carinae) - stars: massive

\section{INTRODUCTION}

The stellar object $\eta$ Carinae (HD 93308; hereafter $\eta$ Car) is one of the most luminous and massive of our Galaxy. Since its Great Eruption in 1843 (Smith \& Frew 2011), it has been frequently observed at a variety of wavelengths. This object has a central source enshrouded by thick ejecta, which, on one hand, precludes a clear view of the central engine, but, on the other hand, displays dynamical and chemical indications of a probable hypernova progenitor (Paczynski 1998; Smith et al. 2007).

The spectrum of $\eta$ Car is rich in low excitation forbidden and permitted lines (Thackeray 1953), as well as high excitation forbidden ones (Damineli et al. 1998, and references therein), which temporarily disappeared in 1948, and again in 1965, 1981, 1987 and 1992. These "spectroscopic events" (Gaviola 1953; Rodgers \& Searle 1967; Thackeray 1967; Zanella et al. 1984) or "low excitation events" (Damineli et al. 1998) were originally believed to be part of S Doradus cycles, commonly seen in other luminous blue variable stars (LBV) similar to $\eta$ Car. This interpretation seemed to be supported by the observations of the He I $\lambda 10830$ line, which reached minimum (Damineli 1996) close to the maximum of the near-infrared light curve (Whitelock et al. 1994). However, the spectroscopic events were demonstrated to be strictly periodic (Damineli et al. 2000), in contrast to the incoherent variations characteristic of S Doradus oscillations.

Periodic minima are in fact observed throughout the electromagnetic spectrum - in doubly ionized forbidden, permitted and UV pumped lines in the optical and UV bands; in near-infrared continuum flux; in X-ray flux; and in $\mathrm{mm}$ and $\mathrm{cm}$ radio flux. The presence of a binary system (Damineli et al. 1997) accounts for the strict periodicity of these minima (Corcoran 2005; Fernandez Lajus et al. 2010; Damineli et al. 2008b). In the binary scenario, the primary star is the most luminous member of the system. It is moderately evolved (in the LBV phase)

\footnotetext{
e-mail to: mairan@astro.iag.usp.br

1 in memoriam

2 Departamento de Física, Universidad de La Serena, Cisternas 1200 Norte, La Serena, Chile

${ }^{3}$ Universities Space Research Association, 10211 Wincopin Circle, Columbia, MD USA 21044

${ }^{4}$ Instituto de Astrofísica de La Plata - CONICET

${ }^{5}$ Dark Cosmology Centre, Niels Bohr Institute, University of Copenhagen, Juliane Maries Vej 30, 2100 Copenhagen Ø, Denmark

${ }^{6} \mathrm{SRON}$, Netherlands Institute for Space Research, 3584 CA, Utrecht, the Netherlands
}

and has a slow $\left(\sim 500 \mathrm{~km} \mathrm{~s}^{-1}\right)$ and dense wind in which the low excitation lines are formed (Hillier et al. 2001, 2006). The companion star is not detected in the spectrum, being less luminous, less massive, hotter and with a fast $\left(\sim 3000 \mathrm{~km} \mathrm{~s}^{-1}\right)$ wind (Corcoran 2005). The orbit is very eccentric and may even be higher than $e=0.9$. At apastron, the secondary star would be placed on our side of the primary, since the Weigelt blobs - which are circumstellar material located $\sim 0.3$ arcsec from the central source at our side of the system (Weigelt \& Ebersberger 1986; Weigelt et al. 1995) - display a high excitation state during most of the orbital cycle (Davidson et al. 1997; Dorland et al. 2004; Smith et al. 2004). The high excitation forbidden lines are absent from the spectrum for a relatively brief time ( $\sim 6$ months) when the system is close to periastron (Damineli et al. 2008a). Moreover, Xray observations indicate that the column density $\left(N_{H}\right)$ is lower toward apastron (Hamaguchi et al. 2007), indicating that the WWC shock cone is opened toward our direction at those phases. However, some authors have suggested different system orientations (Abraham et al. 2005; Soker 2005; Kashi \& Soker 2008; Falceta-Gonçalves \& Abraham 2009). Nevertheless, the binary scenario has gained support from many analyses (Ishibashi et al. 1999; Pittard \& Corcoran 2002; Okazaki et al. 2008; Parkin et al. 2009, 2011).

The suggestion of a wind-wind collision (hereafter WWC; Damineli et al. 1997; Damineli 1997) explains the excess X-ray emission as compared to other binaries containing hot luminous components, especially at energies greater than $2 \mathrm{keV}$. Ishibashi et al. (1999), using the analytical model of Usov (1992), were able to account for the main features in the X-ray emission and for the general behavior of the X-ray light curve, in particular, the increase in the X-ray flux just before periastron. They attributed the rapid drop in X-ray flux at periastron to an increase in the column density $\left(N_{H}\right)$ of the intervening gas from the primary star in the line-of-sight, as the secondary star moved behind the primary. The duration of the X-ray minimum, however, was too long compared to the predictions of the analytical model, and an ad hoc disk around the equator of the primary star was suggested as an additional source of obscuration (Ishibashi et al. 1999). However, Damineli et al. (1999) noted that an eclipse could not produce simultaneously a minimum in X-rays and in the high excitation lines, which are located at different distances and directions relative to our line-of-sight, but which disappear at nearly the same time.

Alternative models assumed transient phenomena 

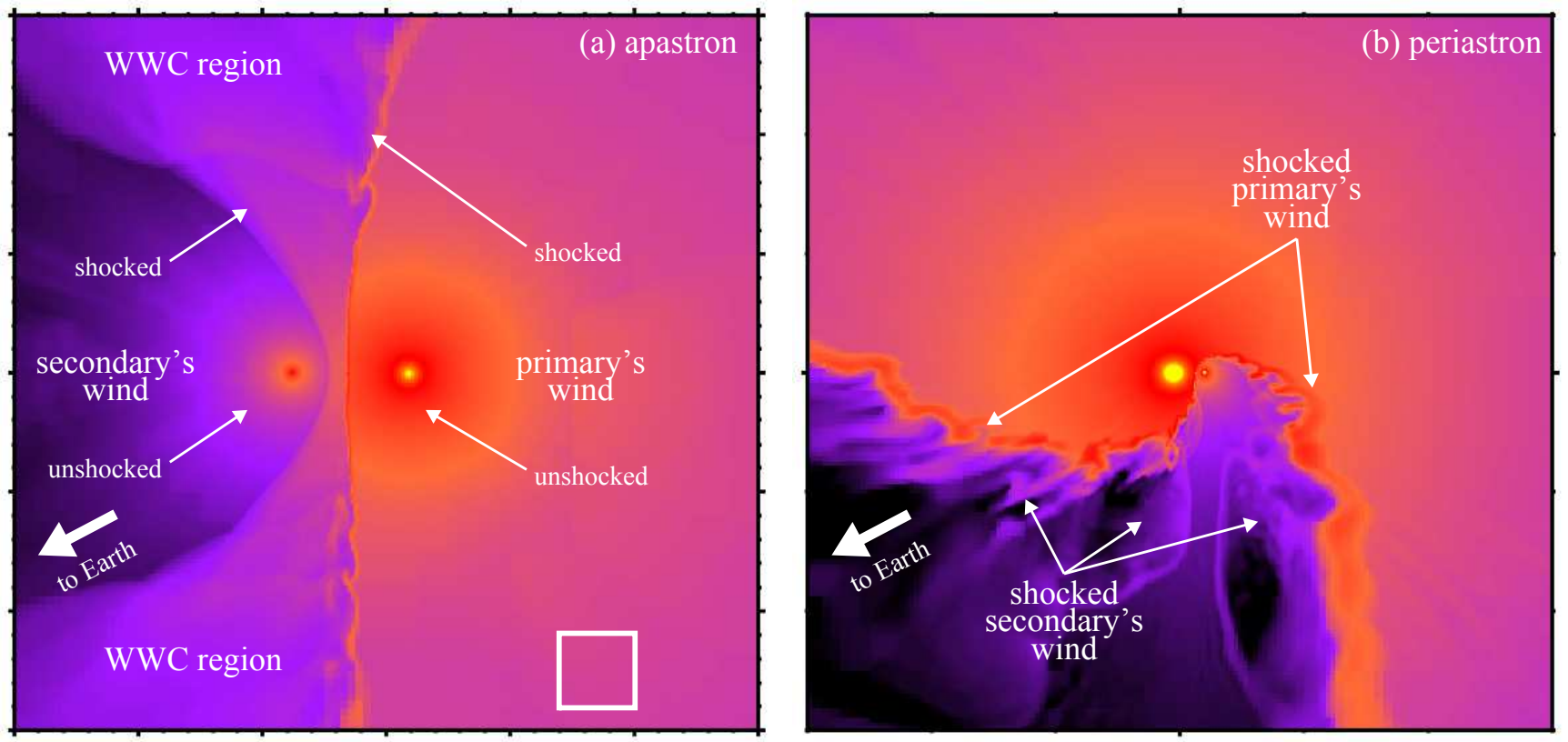

Figure 1. Snapshots of 3D simulations from Parkin et al. (2011): (a) the inner $\pm 15 \times 10^{14} \mathrm{~cm}$ of the system at apastron and (b) the inner $\pm 3 \times 10^{14} \mathrm{~cm}$ of the system at periastron. For comparison, the small box at the bottom of panel (a) has the same dimensions as the entire figure in panel (b). In both panels, the thick arrow indicates the observer's line-of-sight corresponding to a longitude of periastron $\omega=243^{\circ}$ (the observer is located to the lower left).

around periastron to explain the disappearance of X-rays - e.g., shell ejection (Davidson et al. 2005), accretion onto the secondary companion (Soker 2003, 2005; Soker \& Behar 2006; Soker 2007), but all of them face problems when confronted with the whole set of observational data. This is due to the fact that the spectroscopic cycle (i.e. the time interval between two consecutive disappearances of doubly ionized lines or narrow components in He I lines) has two components: a "slow variation" and a "collapse" (Damineli et al. 2008a). Each component affects the cycle in a different way. The "slow variation" component is seen along the whole spectroscopic cycle as a smooth periodic modulation in the radio emission and in the intensity of low-excitation spectral features (e.g. singly ionized lines and the near infrared emission). The minimum of the "slow variation" component is centered at $\phi \sim 0.07^{7}$. The "collapse" component regulates the intensity of the high-energy features (e.g. X-rays, doubly ionized lines) with a minimum centered at $\phi \sim 0.03$. Since the spectroscopic cycle is composed of these two components, any short-lived mechanism (shell ejection, accretion etc.) invoked to explain the "collapse" would not be able to also explain the "slow variation" component.

Modeling the wind-wind collision seems to be a viable option to derive the orbital parameters. Many models to explain the physics of the WWC have been presented, including 2D and 3D numerical simulations (Pittard \& Corcoran 2002; Okazaki et al. 2008; Parkin et al. 2009, 2011). For reference, Fig. 1 shows the WWC from a 3D model Parkin et al. (2011) in which we have identified the main regions and structures discussed in the following sections. Groh et al. (2010) showed that a

7 In this paper, phase $\phi$ is given by $\phi=\left(\mathrm{JD}-\mathrm{JD}_{0}\right) / 2022.7$ where $\mathrm{JD}_{0}=2,452,819.2$ is the time when the narrow component of He I $\lambda 6678$ reached its minimum value during event \#11.
3D numerical smoothed-particle hydrodynamics model (Okazaki et al. 2008) could successfully explain the high velocity absorption component $\left(v \gtrsim 2000 \mathrm{~km} \mathrm{~s}^{-1}\right)$ seen in He I $\lambda 10830$ near periastron. This high-velocity feature requires a large column density of high velocity gas in the line-of-sight, and they derived a periastron longitude $\omega=240^{\circ}-270^{\circ}$ (consistent with an orientation such that the companion is on the near side of the primary at apastron) and orbital inclination $i=40^{\circ}-60^{\circ}$. The He I $\lambda 10830$ line is emitted close to the WWC apex, which revolves very rapidly at periastron. Modeling of the spatially resolved forbidden line emission led to a similar longitude of periastron (Gull et al. 2009, 2011; Madura et al. 2011).

In $\eta$ Car, the detection of He II $\lambda 4686$ emission (Steiner \& Damineli 2004) provided a new tool with which to study the wind-wind collision (Martin et al. 2006; Soker \& Behar 2006; Abraham \& Falceta-Gonçalves 2007). Steiner \& Damineli (2004) found that this emission is present throughout the entire 5.538-yr cycle, but at a low intensity level (equivalent width, $|\mathrm{EW}|<0.1 \AA$ ) for most of the orbit. The intensity of the line rises suddenly by one order of magnitude near $\phi=0$ and then sharply decreases to zero, after which the intensity reaches a lower peak and then declines. The intrinsic luminosity emitted in this spectral line (after correction for interstellar and circumstellar extinction) is greater than the maximum unabsorbed X-ray luminosity in the $2-10 \mathrm{keV}$ band ( $67 L_{\odot}$, Ishibashi et al. 1999). An ultraviolet source with $\sim 10^{4} L_{\odot}$ at energies higher than the $\mathrm{He}^{+}$ionization limit $(54.4 \mathrm{eV})$ is needed to populate the upper level of this line. Keeping in mind that the line formation process for this line has a low efficiency $(\sim 0.3$ per cent, as derived by Martin et al. 2006, and the present paper), then the He II $\lambda 4686$ line reveals a powerful transient energy source in the central region of the system, a peculiar 
state which encouraged us to study this spectral line in greater temporal detail.

The He II $\lambda 4686$ line has been studied in other colliding wind binaries such as HD5980 (Koenigsberger et al. 2010; Breysacher \& François 2000; Moffat et al. 1998), V444 Cygni (Marchenko et al. 1997; Flores et al. 2001), WR127 (de la Chevrotière et al. 2011), and R145 (Schnurr et al. 2009). In those systems, it is not easy to isolate the contribution from the WWC, since the stellar winds themselves make an important contribution to the emission (e.g. in the case of V444 Cygni, the WWC region contributes only $\sim 10$ per cent of the He II $\lambda 4686$ emission; Marchenko et al. 1997; Flores et al. 2001). Since the line emission is very faint far from periastron in $\eta$ Car, the emission around periastron can be associated entirely with the WWC. There are other examples of spectral lines being formed exclusively in the WWC in other $\mathrm{WC}+\mathrm{O}$ binaries, like the $\mathrm{C}$ III $\lambda 5696$ excess in WR140 (Fahed et al. 2011), Br22 (Bartzakos et al. 2001), and $\theta$ Mus (Hill et al. 2002).

There are only two plausible regions in the system that could produce the observed He II $\lambda 4686$ emission near periastron in $\eta$ Car: the shocked stellar wind of the primary or that of the secondary star. The latter seems insufficient to produce enough $\mathrm{He}^{++}$ions for recombination due to its high temperature and low density (Martin et al. 2006) whereas the former seems to have the most favorable physical conditions (e.g. density, temperature and velocity field) for that process to occur.

Martin et al. (2006) showed that UV photons with $h \nu>13.6 \mathrm{eV}$ could ionize $\mathrm{He}^{+}$excited to the $\mathrm{n}=2$ level to $\mathrm{He}^{++}$and that Hydrogen-Ly $\alpha$ could also excite $\mathrm{He}^{+}$ ions from the $n=2$ to $n=4$ level, which would produce the He II $\lambda 4686$ line via electronic cascade. This could happen along the WWC region, where the gas has a relatively low temperature $\left(T<10^{5} \mathrm{~K}\right)$. Therefore, the He II $\lambda 4686$ emission would be somewhat connected to the hard X-ray source, but not spatially coincident with it, and the temporal behavior of both emissions should be similar. Also, the secondary star evacuates a cavity in the primary's wind near periastron, which could provide an additional source of UV photons from the inner wind of the primary. However, this emission would probably deliver much less energy than is required to power the He II $\lambda 4686$ line: $|\mathrm{EW}| \sim 0.1 \AA$ (Martin et al. 2006).

The main goal of our observing campaign is to constrain the physical emission mechanism and to determine the particular location of the He II $\lambda 4686$ emitting region. The present paper is organized as follows. In $\S 2$, the observations and data processing are presented. In $\S 3$, the adopted methodology for measuring the equivalent width and velocities of the He II $\lambda 4686$ line are shown. In $\S 4$ we present the results and in $\S 5$ the discussion. Finally, our main conclusions are shown in $\S 6$.

\section{OBSERVATIONS AND DATA REDUCTION}

We used the terminology of Groh \& Damineli (2004) in naming the cycles: \#1 starts in 1948 (the first low excitation event recorded by Gaviola 1953) and the last one in 2009, which is cycle \#12. In this paper, we use the term "low excitation event" (or just "event") to refer to the disappearance of high excitation spectral lines, which are used to define the spectroscopic cycle. We also used the term "spectroscopic cycle" to refer to the time interval between two consecutive low excitation events (5.538 yr; Damineli et al. 2008a). The time of periastron passage is not known so we assign $\phi=0$ to the minimum of the narrow component of the He I $\lambda 6678$ emission line, which occurred on $\mathrm{JD}=2,452,819,2$ for event \#11 (Damineli et al. 2008b). We follow those authors also for the period length $(\mathrm{P}=2022.7 \pm 1.3$ days $)$, determined by minimization of residuals when folding light curves around the minimum in cycles \#9, \#10 and \#11. This period is very robust, since it is based on the best sampled light curves, including the X-ray, optical, and nearinfrared broad band light curves, and from variations in the strengths of several spectral lines.

\subsection{Event \#12 (2009.0 minimum)}

The 2009.0 minimum was spectroscopically monitored using five astrophysical observatories: SOAR (Southern Astrophysical Research Telescope/Chile), OPD (Observatório Pico dos Dias/Brazil), ESO (European Southern Observatory/Chile), CASLEO (Complejo Astronómico El Leoncito/Argentina), and LCO (Las Campanas Observatory/Chile). All the relevant data about the results presented in this paper are listed in Table 1.

Daily monitoring of the optical spectrum of $\eta$ Car was carried out at SOAR from 2008 December 21 through 2009 January 23. After that, data were obtained once per month. The Goodman optical spectrograph was used to obtain spectra with spectral resolving power $R \sim 2800$ (3 pixels) in the range $\lambda \lambda 3500-6850 \AA$. All the observations were performed using the 0.45 arcsec-wide slit aligned with the parallactic angle. For each visit, a hot standard star (HD 303308; O4.5 V((fc)); Walborn et al. 2010; Sota et al. 2011) was observed to correct for telluric lines and for low-frequency variations in the detector response along the spectral dimension. Processing, reduction, and calibration of the SOAR data were done using the standard long-slit spectroscopic packages of IRAF ${ }^{8}$ (Tody 1993). The 1D spectrum was extracted using the IRAF package DOSLIT and the spatial extraction aperture was about 1.5 arcsec for all the SOAR spectra. Performing extractions with apertures as large as 4.5 arcsec does not introduce any significant change in either the He II $\lambda 4686$ line profile or in the measured equivalent widths. Before or after each set of observations of $\eta$ Car, 2 exposures of an $\mathrm{HgAr} / \mathrm{CuAr}$ lamp were obtained for wavelength calibration.

The FEROS (fiber-fed extended-range echelle spectrograph) data were obtained at the $2.2 \mathrm{~m}$ telescope (Max Planck Institute). FEROS delivers data with resolving power $R \sim 48000$ ( 2 pixels) and spectral coverage from 3700 to $8600 \AA$ in a single exposure. It uses two fibers with diameter of 2 arcsec separated by 2.9 arcmin. The processing, reduction, and calibration were performed using the FEROS standard reduction pipeline, which is described in Kaufer et al. (1999).

Spectra were also measured at the Coudé focus of the $1.60 \mathrm{~m}$ telescope of the Pico dos Dias Observatory (OPD/Brazil), with $R \sim 10000$ (2 pixels). For each visit, a hot star ( $\theta$ Car; B0.2 V; Hubrig et al. 2008; Nazé

\footnotetext{
8 IRAF is distributed by the National Optical Astronomy Observatories, which are operated by the Association of Universities for Research in Astronomy, Inc., under cooperative agreement with the National Science Foundation.
} 


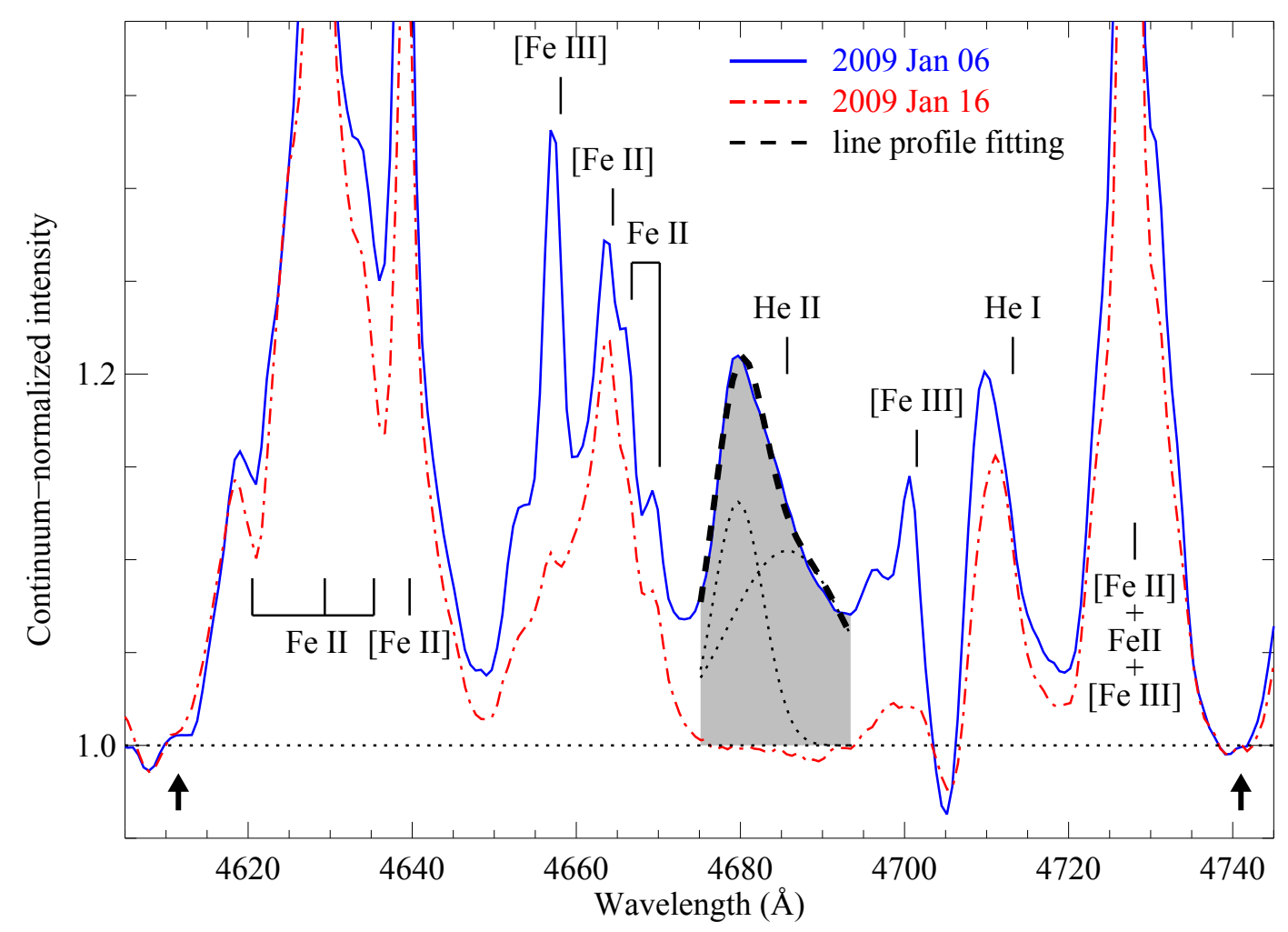

Figure 2. He II $\lambda 4686$ at minimum (dash-dotted red line) and maximum (solid blue line). The observed line profile was fitted by two gaussian components (dotted lines) and the result (dashed line) was used to measure the radial velocity. The two arrows indicate the regions adopted as continuum level for the He II $\lambda 4686$ emission line. The equivalent width was derived by direct integration under the line profile (gray shaded area).

\& Rauw 2008) was also observed to correct the shape of the continuum for the variations in the instrument's response. The size of the extraction aperture for the OPD data was about 2 arcsec. A ThAr spectrum was obtained immediately preceding or following the $\eta$ Car observations for wavelength calibration.

We also obtained spectra at the $6.5 \mathrm{~m}$ Magellan telescope located at Las Campanas Observatory, using the échelle spectrograph MIKE, with $R \sim 25000$ ( 2 pixels) in the blue region. MIKE's data processing was performed by cutting from the raw échelle images only the orders that contained the He II $\lambda 4686$ line and its continuum region (orders from 75 to 78 ). Using this approach, we could process, reduce, and calibrate the data using typical IRAF échelle routines. For those data, we used the spectrum of a hot standard star (HD 303308, the same one used for the SOAR dataset) for a first rectification of the low-frequency variations along the spectral dimension.

Spectroscopic data were also obtained with the EBASIM and REOSC spectrographs at the CASLEO Observatory $2.15 \mathrm{~m}$ telescope. Both are échelle spectrographs with $R \sim 42000$ and $R \sim 12000$, respectively. The data reduction and processing were performed using standard IRAF tasks for échelle data.

The signal-to-noise ratio of the data from the 2009.0 campaign was always higher than 100 for all these observations, frequently reaching $\mathrm{S} / \mathrm{N} \sim 250$.

\subsection{Previous events: \#9 (1992.5), \#10 (1998.0) and \#11 (2003.5)}

The data from previous events shown in the present paper were obtained at the Coudé focus of OPD and with the FLASH/HEROS spectrograph at the ESO $0.52 \mathrm{~m}$ telescope (events \#9 and \#11; we would like to remark that the ESO dataset for event \#9, used in the present work, was wrongly assigned to OPD in Steiner \& Damineli 2004). Spectra were also taken on 1997 November and December, and 1998 February and March, at the Coudé focus of the $1.9 \mathrm{~m}$ telescope of the Mount Stromlo and Siding Spring Observatory (MSSSO) with $R \sim 60000$ in the range from 4346 to $4935 \AA$ (McGregor et al. 1999).

\section{MEASUREMENT METHODOLOGY FOR THE He II $\lambda 4686$} LINE

In order to have a homogeneous dataset and to minimize systematic errors, we first identified the region to be considered as the He II $\lambda 4686$ reference continuum. Fig. 2 illustrates the regions we adopted for the integration and continuum, which were set to a small wavelength interval (3 $\AA$ wide) near $4610 \AA$ and $4740 \AA$. Since the spectra were previously flattened using the spectrum of a hot star, the continuum in this range is well represented by a linear fit, and the equivalent width was measured by direct integration of the line profile in the range from 4675 to $4694 \AA$. The regions defined in this work for the line integration and continuum are very similar to those adopted by Martin et al. (2006) using Hubble Space Tele- 


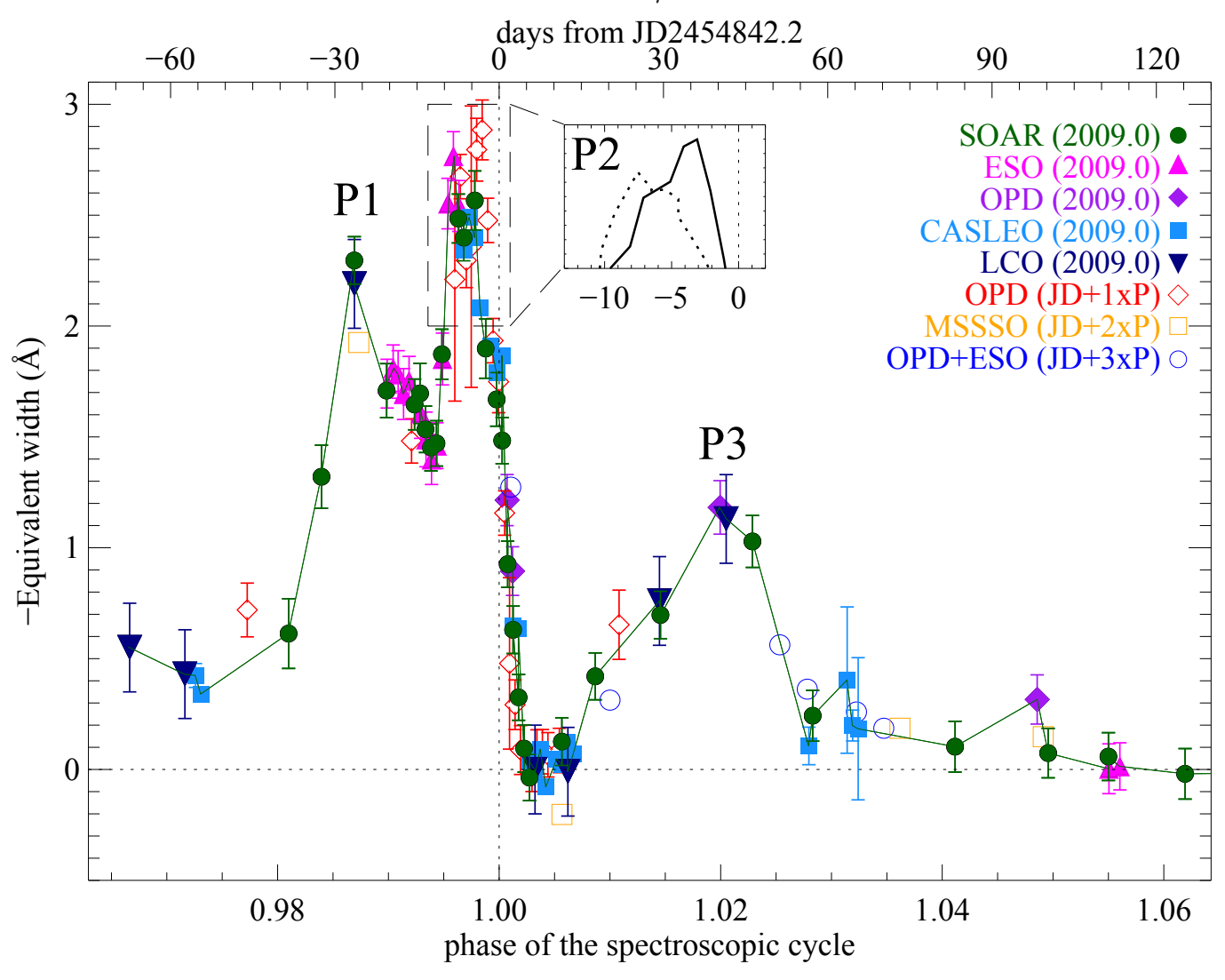

Figure 3. Equivalent width of He II $\lambda 4686$ for the 2009.0 event (filled symbols) and for past events (open symbols) taken at different observatories. Data from the 3 previous events are over plotted as empty symbols, and folded with a period of $\mathrm{P}=2022.7$ days. Each local peak discussed in the text are labeled in this figure as P1, P2 and P3. The inset panel shows the mean value of the equivalent width around peak P2 for event \#11 (solid line) and \#12 (dotted line) and illustrates that the peaks do not seem to be phase-locked. Indeed, for event \#12, P2 occurred $~ 4$ days before the phase observed for event \#11.

scope $(H S T)$ data.

In order to have a robust determination of the radial velocity of the line peak, we used a two gaussian model to fit the integration region of the line profile, since a single gaussian profile did not reproduce the line profile over the integration region across the low excitation event, especially when the equivalent width reaches its maximum value near $\phi=0$. Fig. 2 shows the two gaussian components used to fit the He II $\lambda 4686$ line profile. The resulting 2-component fit was then used to find the wavelength at which the line profile reaches its peak intensity. Note that, since we are interested only in the radial velocity of the line peak, the line wings, which are much more extended, do not influence the derived velocity of the line peak. All of the radial velocities measured in the present paper are in the heliocentric frame.

The main source of uncertainties in the methodology for measuring the equivalent width is the normalization of the continuum. Data obtained in échelle mode suffer from order overlap and corrections for this overlap are crucial for tracking the real stellar continuum. We normalized the echelle spectra in two steps. First, we divided the flat-fielded spectrum by the continuum of a hot star, then we applied a parabolic fit to finally rectify the continuum. We rejected data in which the rectified continuum showed low-frequency variations greater than 1 per cent.

As mentioned previously, the wavelength calibration was obtained using comparison lamps. It is impossi- ble to ascribe a single representative error to the entire observational dataset because each instrument has its own characteristics, reduction procedure and calibration methods. However, we checked the reliability of our procedure by comparing multiple observations taken on different telescopes in the same night. Discrepancies in velocity are smaller than $50 \mathrm{~km} \mathrm{~s}^{-1}$. Equivalent width measures from different telescopes at similar phases are also in quite good agreement.

We claim that our set of He II $\lambda 4686$ spectra is the most accurate reported so far. It has the best time sampling over the last two events $(\# 11=2003.5$ and $\# 12$ $=2009.0)$ in addition to some data for cycles \#9 and \#10. Also, ground based spectra - because they are spatially unresolved - are very robust for time variation studies, since most of the emission comes from $\sim 1$ arcsec around the central source, which is blurred by the seeing so that the ground based slit is always sampling the relevant emitting region.

The absence of He II $\lambda 4686$ emission on MJD 52852 reported by Martin et al. (2006) might be caused by a bad datum or a real momentary fading of the line. Despite the fact that we cannot directly compare the results because we do not have simultaneous ground-based data, we call attention to the fact that our monitoring along 3 cycle (\#9, \#11 and \#12) always show this line in emission, confirming that P3 is a repeatable feature. Anyway, this point can be clarified in the next event (in 2014.6). 


\section{RESULTS}

The observed He II $\lambda 4686$ equivalent width curve is shown in Fig. 3, which presents data for the last four events (\#9, \#10, \#11 and \#12) using the following ephemerides:

$$
\mathrm{JD}=\mathrm{JD}_{0}+2022.7(E-11),
$$

where $\mathrm{JD}_{0}=2,452,819.2$ (time of minimum intensity of He I $\lambda 6678$; Damineli et al. 2008b) and $E$ is the cycle + phase count. We focused on a time interval ranging from 2 months before up to 4 months after $\phi=0$. In this paper, we use the word "day" as a reference to the number of days before (indicated by a minus sign) or after (plus sign) $\phi=0$ for each cycle.

The 2003.5 spectra used to measure the equivalent width and radial velocities shown in this paper are the same as those presented in Steiner \& Damineli (2004) but reduced according to the procedures described in $\S 2$ in order to keep the whole set of data as homogeneous as possible. Fig. 4 shows that He II $\lambda 4686$ was detected with $\mathrm{EW} \sim-0.1 \AA$ in a spectrum taken at the OPD telescope on 2008 May 08, about 8 months before the low excitation event, and also at the same strength in another spectrum taken 17 months after $\phi=0$, on 2010 June 20 (using the same telescope and instrumental configuration). Another spectrum, taken on 2011 July 11, at $\phi=12.451$ - almost apastron - also shows a very faint emission, which may be He II $\lambda 4686$. These observations $^{9}$ suggest that He II $\lambda 4686$ is present, except for a 5 day interval after the start of the spectroscopic event, along the whole 5.538-yr cycle (Steiner \& Damineli 2004), though Martin et al. (2006) claimed that this line is not present at any phase outside the low excitation event. The observed continuum-normalized time series spectrum of $\eta$ Car in the region $\lambda \lambda 4660-4710$ is shown in Fig. 5. In that figure, we interpolated the spectra in phase to show the temporal evolution of the He II $\lambda 4686$ emission line. Notice that Fig. 5 mainly reflects the progression of the cycle \#11 to \#12 since data are sparse for other cycles.

A major feature in the intensity curve for event \#12 is the presence of 3 local maxima (labeled in Fig. 3 and 5 as P1, P2 and P3). The first local maximum, P1, occurred about day -26 , and showed a conspicuous peak with $\mathrm{EW}=-2.30 \AA$. The second local maximum, P2, occurred between day -10 and -5 , and during this time interval, the mean equivalent width was about $-2.5 \AA$. After day -5 , the equivalent width of the line rapidly decreased to zero and it completely disappeared by day +5 . From day +5 to +10 the equivalent width was zero. After day +10 it started to increase again, to a local maximum value of $-1.2 \AA$ (P3) at day +40 , and then decreased to $-0.1 \AA$ after day +115 . The rate of decrease of the EW just before minimum was about $-0.3 \AA$ per day and the $e$-folding time scale in the fading phase (after P2) was $\sim 2$ days.

There are only a few sparse data near the low excitation event for cycles earlier than cycle \#12 (except for cycle \#11 in 2003.5, as shown in Steiner \& Damineli 2004); despite this we can see that the general behavior

9 Notice that none of them are included in Fig. 3 because they are far from the low excitation event. of the equivalent width of the He II $\lambda 4686$ line repeats faithfully from cycle to cycle. Peaks P1 and P2, however, do not seem to be phase-locked: for event \#12, P2 (the best time sampled peak) occurred $\sim 4$ days before the phase in which it was detected in event \#11 (see inset in Fig. 3). Although there were no data in the earlier cycles corresponding to the phase of the peak P3 maximum, the behavior of the line equivalent widths observed in the 1992.5 and 2003.5 events (see Fig. 3) suggests that P3 is a phase-locked feature.

\subsection{He II $\lambda 4686$ luminosity}

The intrinsic line luminosity $L^{0}$ of He II $\lambda 4686$ is given by

$$
L^{0}=4 \pi D^{2} f_{\lambda 4686}^{0} \mathrm{EW}_{\text {He II } \lambda 4686},
$$

where $D$ is the distance to the system $(2.3 \mathrm{kpc})$, $\mathrm{EW}_{\mathrm{He} \text { II } \lambda 4686}$ is the equivalent width of the He II $\lambda 4686$ line (measured in the present work), and $f_{\lambda 4686}^{0}$ is the intrinsic flux from the central source near $\lambda=4686 \AA$. We assume that $f_{\lambda 4686}^{0} \approx f_{B}^{0}$, where $f_{B}^{0}$ is the dereddened $B$-band flux from the source. The observed $B$-band flux $\left(f_{\mathrm{B}}^{\text {obs }}\right)$ is derived from observed $B$-band magnitudes following this relation:

$$
f_{\mathrm{B}}^{\mathrm{obs}}=6.5 \times 10^{-10} \times 10^{-0.4 B},
$$

where $B$ is the observed $B$-band brightness of the central source, as obtained by Fernandez Lajus et al. (2009). It is important to remark that Fernandez Lajus et al. (2009) used a $12^{\prime \prime}$ radius aperture, which includes the entire Homunculus - an important source of scattered light. In order to match the $1^{\prime \prime}$ region sampled by the ground based spectroscopy, we used a $B$ band image to determine the magnitude difference between a $1^{\prime \prime}$ circular aperture centered on the star and the $12^{\prime \prime}$ aperture used for the rest of the photometry. This showed that the $B$ band magnitudes reported by Fernandez Lajus et al. (2009) needed to be fainter by +1.6 mag to match the spectral aperture. The dereddened flux at $4686 \AA$ is then given by

$$
f_{\lambda 4686}^{0} \approx f_{B}^{0}=f_{B}^{\text {obs }} \times 10^{0.4 A_{B}},
$$

where $A_{B}$ is the extinction in the $B$ band. However, since we have no direct measurement of $A_{B}$, we adopt the usual value for $A_{V}$ (Davidson et al. 1995; Hillier et al. 2001) to obtain $A_{B}$ using the following linear relation from Cardelli et al. (1989):

$$
\frac{A_{B}}{A_{V}}=a(\lambda)+\frac{b(\lambda)}{R_{V}},
$$

where $a(\lambda)$ and $b(\lambda)$ are constants which depends on the wavelength and, in the present work, need to be evaluated in the $B$ band region $(\lambda=4500 \AA)$.

The secular brightening of $\eta$ Car has been interpreted as a decrease in extinction (see van Genderen et al. 2006), probably due to a expansion in the circumstellar shell, so we need to estimate the decrease of $A_{V}$ with time. In 1998, in the line of sight to the central source there were about $A_{V}=7$ magnitudes of total extinction (interstellar and circumstellar; Hillier et al. 2001) from which $\sim 2$ magnitudes were due to gray extinction associated with large grains around the central source. Based on this result, in early 2006 the estimated extinction was $A_{V}$ 


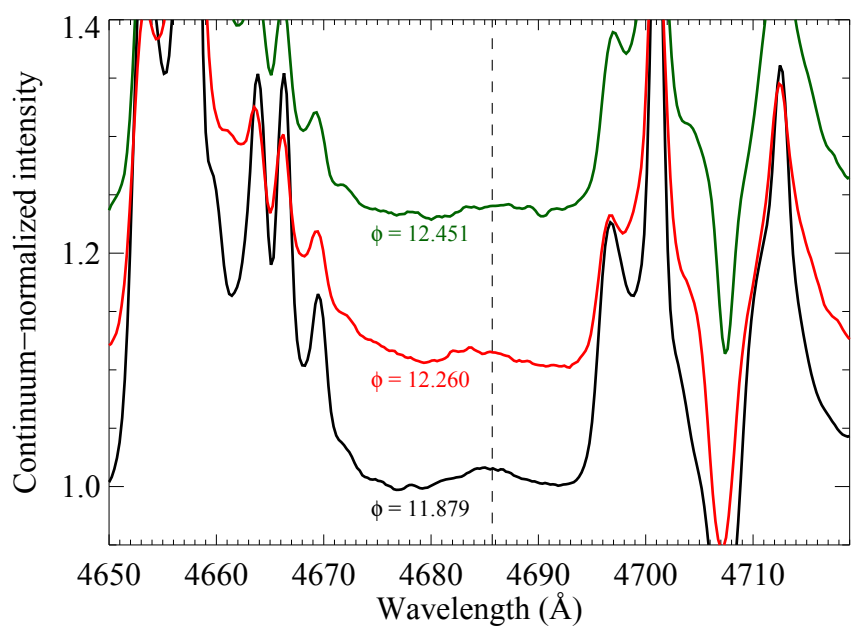

Figure 4. Spectra of $\eta$ Car taken on 2008 May $10(\phi=11.879$; black line), 2010 Jun 20 ( $\phi=12.260$; red line), and 2011 July $11(\phi=12.451)$, showing the presence of He II $\lambda 4686$ at 8 months before, 17 months after and 31 months after the event, respectively (spectra taken at the OPD/Coudé). The corresponding phase of the spectroscopic cycle is also indicated. He II $\lambda 4686$ far from periastron is present at faint level, $\mathrm{EW}<-0.1 \AA$

$=5.7$ (van Genderen et al. 2006). From 2003 to 2009, the average rate of increase in the brightness of the central source was $-0.06 \mathrm{mag} \mathrm{yr}^{-1}$. Therefore, the total extinction to the central source in 2003.5 was $A_{V}=5.9$, and in 2009.0, $A_{V}=5.5$. We do not correct for the scattering in the primary's wind, which should contribute to a cyclic variability in $A_{V}$.

In order to transform from $A_{V}$ to $A_{B}$, we used eq. (5), with $R_{V}=4.9$ (Hillier et al. 2001; van Genderen et al. 2006). Hence, with the photometry in the $B$ band and the total extinction $A_{B}$, we were able to calculate the intrinsic flux $f_{\lambda 4686}^{0}$ and thus the intrinsic luminosity of the He II $\lambda 4686$ emission line from eq. (2).

The results are shown in Fig. 6a, where the intrinsic line luminosities are shown - as well as the photon fluxes - for two events: 2003.5 and 2009.0. The line luminosity of the 2003.5 event was higher than that of 2009.0 (for a given phase): the peak values for the intrinsic line luminosity were, respectively, $310 L_{\odot}$ (corresponding to $1.8 \times 10^{47}$ He II $\lambda 4686$ photons per second) and $250 L_{\odot}$ $\left(=1.4 \times 10^{47}\right.$ He II $\lambda 4686$ photons per second). We cannot claim that there is a real difference in the intrinsic luminosity, since the extinction might have varied in shorter time scales than we adopted. These luminosities are probably lower limits to the true line luminosity since the adopted correction for extinction is only a simple estimate, and because obscuration by the wind of the primary star could also be significant.

\subsection{Radial velocities}

During most of the spectroscopic cycle, the radial velocity of the line peak was close to zero. Near the low excitation event, there were two regimes for the rate of change in the radial velocity (shaded areas $\mathrm{R} 1$ and $\mathrm{R} 2$ in Figure $6 \mathrm{~b})$. Region $\mathrm{R} 1$ is near day -90 when the radial velocity of the line peak changed at a daily rate of about $-2 \mathrm{~km} \mathrm{~s}^{-1}$ (shifting from $-50 \mathrm{~km} \mathrm{~s}^{-1}$ on day -67 to about $-150 \mathrm{~km} \mathrm{~s}^{-1}$ on day -17$)$. The first peak in the equivalent width (P1) occurred during this time interval,

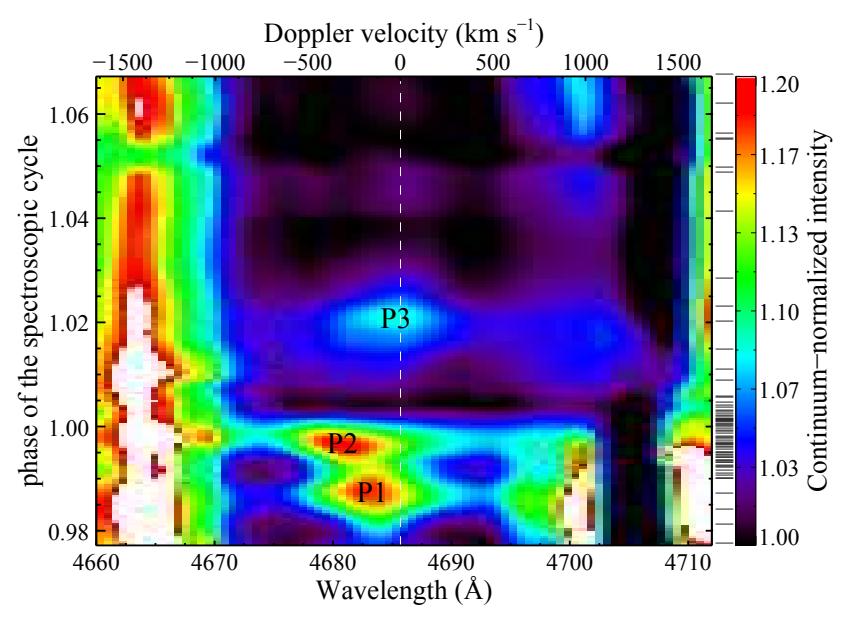

Figure 5. Folded time series spectra of the He II $\lambda 4686$ line profile along the 4 last low excitation events. The continuum-normalized intensity of the spectra is color-coded between 0 and 20 per cent of the continuum level for visualization purposes. The vertical dashed line marks the rest wavelength of the He II $\lambda 4686$ line emission. The tick marks located on the right, between the color bar and the image, indicate the phases when spectra were obtained.

on day -26 . The second regime (R2) started on day -17 and was observed until the line completely disappeared, on day +6 . During this time interval, the rate of change in the radial velocity of the line peak was almost seven times greater than in the previous interval: it started at $-150 \mathrm{~km} \mathrm{~s}^{-1}$ on day -17 , and reached $-450 \mathrm{~km} \mathrm{~s}^{-1}$ on day +5 , a change of nearly $14 \mathrm{~km} \mathrm{~s}^{-1}$ per day. Between day +6 and +15 the line could not be measured.

The general behavior of the He II $\lambda 4686$ radial velocity suggests that this line is formed in the WWC region, in the interval from 2 months before to 2 months after the minimum. This is supported by the observed velocity of the X-ray line S XVI Ly $\alpha$ (Figure 6b; Henley et al. 2008). The time variations in radial velocity for both lines are similar: they become more blue-shifted near $\phi=0$ and then shift to projected velocities near zero. However, the velocity of the S XVI Ly $\alpha$ line just prior to the low-excitation event is more than a factor of 2 larger than that of He II $\lambda 4686$ line. This is surprising, since we expect the S XVI Ly $\alpha$ line to be emitted close to the apex, where the flow velocity is slower than it is downstream along the WWC region. On the other hand, near $\phi=0$, the WWC gets very distorted and unstable, which could result in a mixed contribution from different regions.

The interpretation of the He II $\lambda 4686$ radial velocity curve is not as simple as in other colliding wind systems because of the severe Coriolis distortion of the shock near periastron passage. As the stars approach periastron, the leading and the trailing arms of the shock are subject to different drag forces and the cone is heavily distorted. In addition, the inner zones, close to the apex, revolves fast in a spiraling pattern. Because of these complications, the projected radial velocities of the gas which produces the observable emission cannot be modeled using a simpler, rigid-cone geometry as has been used effectively for other colliding wind binary systems.

It is interesting to notice that the radial velocity of the peak of the He II $\lambda 4686$ line emission shifts to the blue almost at the same time as the He I $\lambda 10830$ P Cyg absorption shows a high-velocity absorption component 

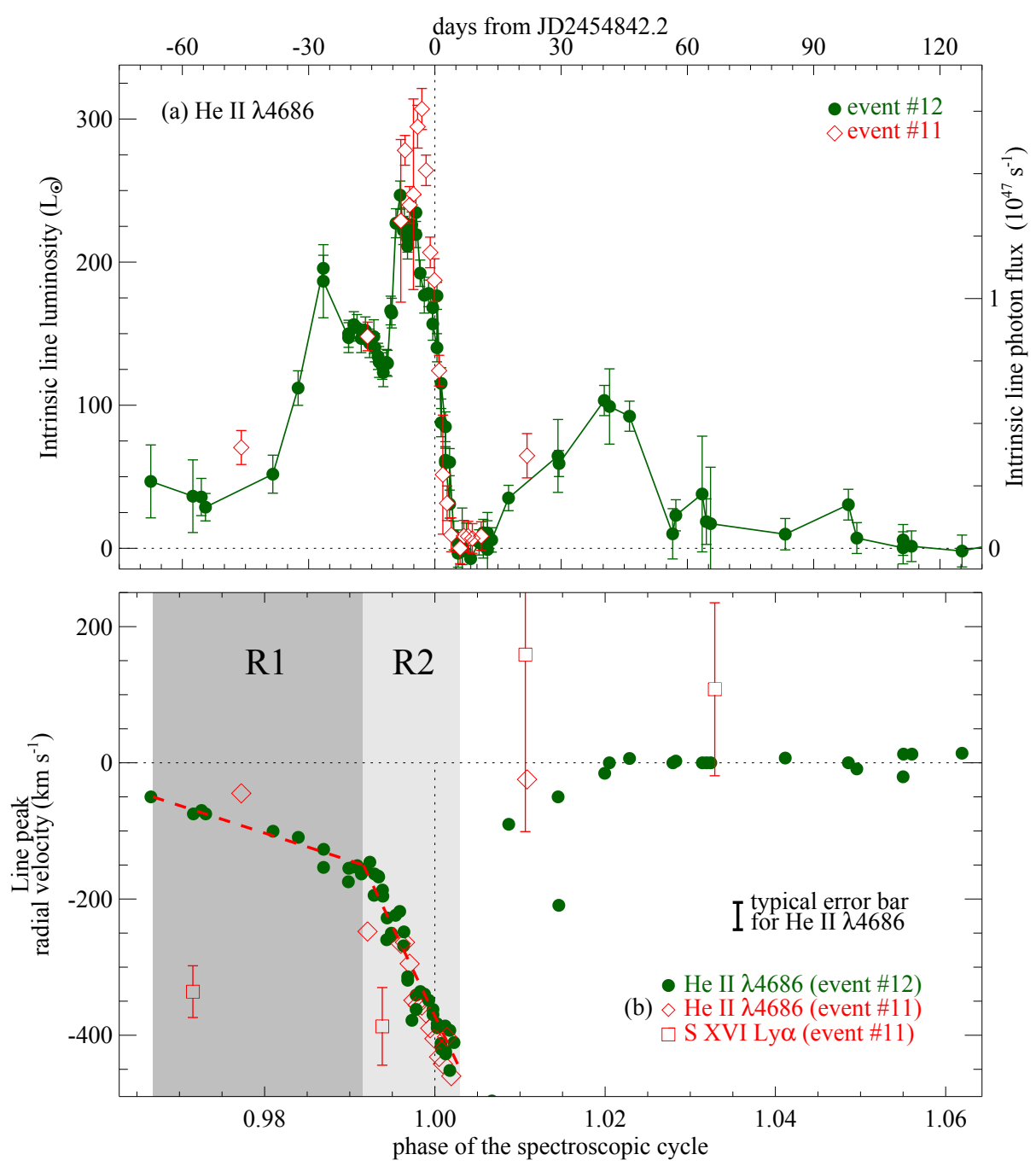

Figure 6. (a) Lower limit for the intrinsic luminosity of the He II $\lambda 4686$ line for 2009.0 (filled green circles) superimposed on the 2003.5 event (empty red diamonds) folded with a period of $\mathrm{P}=2022.7$ days. (b) Radial velocity of the peak of the He II $\lambda 4686$ line for both events and the observed velocity of the S XVI Ly $\alpha$ for the 2003.5 event (red squares; data extracted from Henley et al. 2008). The shaded areas indicate the two different regimes (R1 and R2) for the rate of change in the radial velocity curve. The abscissa in panel (a) is plotted in the same time scale as in (b).

bluer than $-900 \mathrm{~km} \mathrm{~s}^{-1}$ (Groh et al. 2010). Since the velocity field of the WWC region has a complex pattern close to periastron, we expect to see a wide range of velocities and this is in fact present in the large FWHM of those two lines. However, this interpretation may be too simple, since the absorption component samples a very limited region in our line of sight while the emission receives contributions from many other regions.

We should only see high positive velocities coming from the WWC region when it is pointing away from us, during periastron passage. Due to the high orbital eccentricity, the WWC cone does not rotate much until just before periastron passage, which means that the time spent by the WWC cone pointing away from the observer is very short. From Fig. 6, it is evident that the maximum negative radial velocity occurs for P2, which is detected between day -5 and -10 . Therefore, based on symmetry arguments for the motion of the secondary around the primary, the maximum positive radial velocity should occur somewhere between day +5 and +10 , which corresponds to the interval when the He II $\lambda 4686$ line is absent. Note, however, that this interval coincidently may occur at the same time as the disruption of the WWC region.

The radial velocity of the peak of the He II $\lambda 4686$ line profile showed the same behavior for events \#11 and \#12: it reached up to $-450 \mathrm{~km} \mathrm{~s}^{-1}$ just before the minimum and completely vanished during 5 days (from day +5 to +10$)$. After that, the peak was detected again with a radial velocity of $-100 \mathrm{~km} \mathrm{~s}^{-1}$, which changed to about zero $\sim 40$ days after $\phi=0$.

\subsection{Correlation between He II $\lambda 4686$ and X-rays}

During the 2009.0 low excitation event, after shifting the $\mathrm{X}$-ray light curves in time by +16.5 days, the regime of fast decrease in the X-ray flux began approximately at the same time as the decrease in the He II $\lambda 4686$ equivalent width (see Fig. 7a). The major peaks in the X-ray flux preceding the event also seem to have counterparts in the He II $\lambda 4686$ equivalent width peaks. The 2003.5 observations did not show clearly the same correlation between the X-ray flares and He II $\lambda 4686$ peaks (see Fig- 

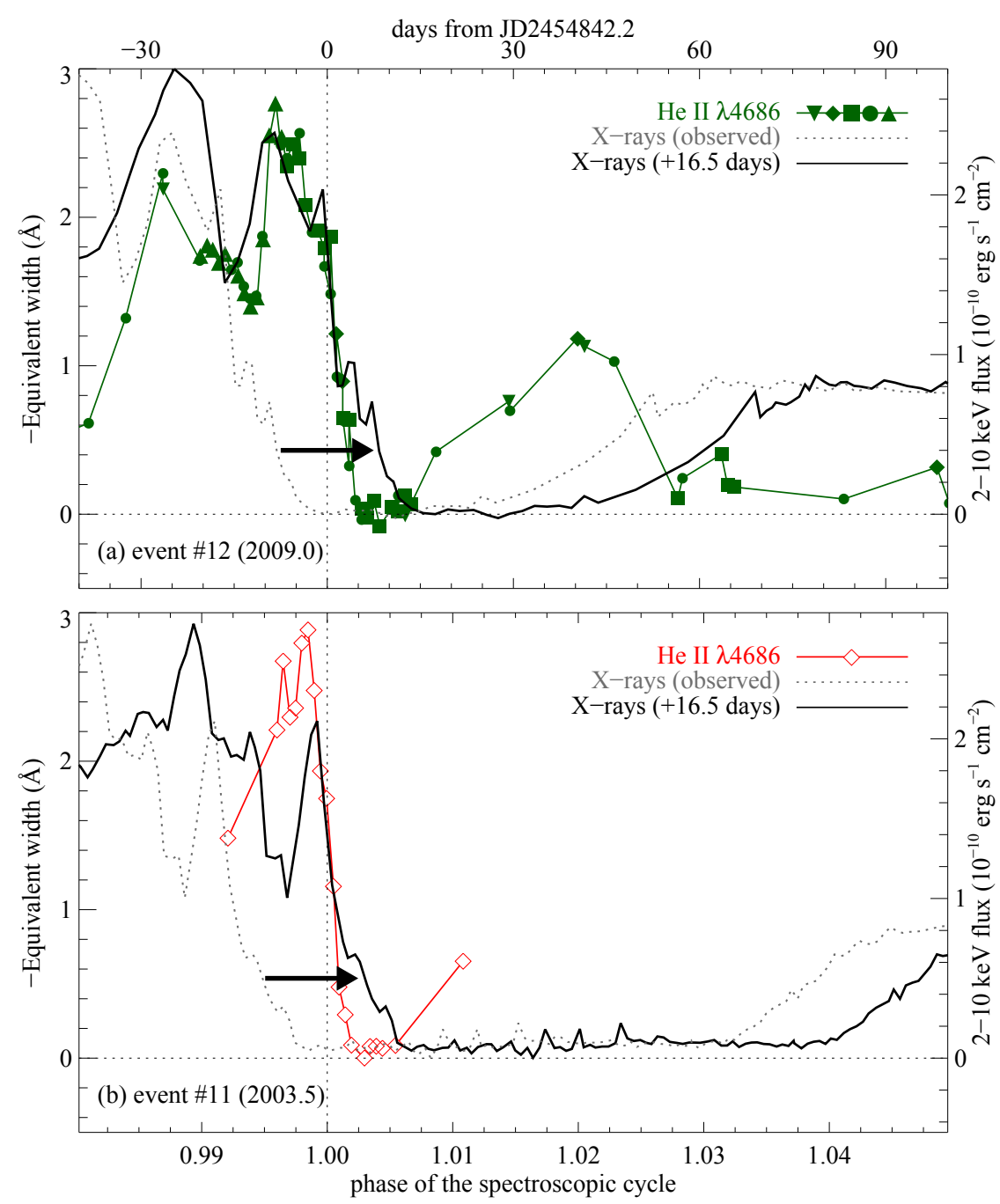

Figure 7. Equivalent width curve of He II $\lambda 4686$ (symbols) over plotted with the $2-10 \mathrm{keV}$ X-ray flux. In both panels, the observed X-ray flux is illustrated by the dotted gray line (from Corcoran 2005), while the solid black line was shifted by +16.5 days. Panel (a) is for cycle \#12 and (b) is for \#11.

ure $7 \mathrm{~b}$ ), possibly because of sparser monitoring and/or lower quality He II $\lambda 4686$ data. This suggests a possible connection between the X-ray and the He II $\lambda 4686$ behavior. The delay of 16.5 days could be interpreted as the flow time of the shocked material, from the region where X-rays are created (in the WWC apex) to that where the temperature has dropped below $6 \times 10^{5} \mathrm{~K}$, allowing for the recombination of $\mathrm{He}^{++}$into $\mathrm{He}^{+}$. If so, assuming a flow velocity of $500 \mathrm{~km} \mathrm{~s}^{-1}$ for the shocked material (terminal velocity of the primary's wind; Hillier et al. 2001; Pittard \& Corcoran 2002), then the bulk of the He II $\lambda 4686$ emission should come from a region $4-5$ AU from the apex, which is consistent with the results of 3D models (Parkin et al. 2011), which predict a temperature of $\sim 10^{5} \mathrm{~K}$ in the primary's shocked stellar wind at that distance from the apex.

It is interesting to notice that the X-ray light curve of WR $140^{10}$ is similar to that of $\eta$ Car in the sense

10 This system is the archetypal of the colliding wind binaries and is composed of a WC7+O4-5 binary in a highly eccentric ( 0.896), long period ( $\sim 2896.5$ days) orbit (see Williams et al. 1990; White \& Becker 1995; Marchenko et al. 2003; Fahed et al. 2011). that there is a gradual increase until a maximum, followed by a sharp decrease (Williams 2011; Corcoran et al. 2011). The main difference between these X-ray light curves is the duration of the X-ray minimum, which is much longer for $\eta$ Car (perhaps caused by the collapse of the WWC region, as discussed in the next section). Another important difference is the presence of X-rays flares in $\eta$ Car when approaching periastron, which are not seen in WR140; this might suggest that in the stellar wind of the WC7 primary component of WR 140, the clumps are smaller than those in the LBV primary star in $\eta$ Car (Moffat \& Corcoran 2009).

\subsection{He II $\lambda 4686$ far from periastron}

The faint emission seen along most of the orbit could be emitted in the WWC itself, since far from periastron, the density in the shock is smaller and the efficiency of He II $\lambda 4686$ pumping is lower. However, since cooling is less efficient at lower density, the emission would occur farther from the apex, as compared to the strong emission at periastron. The speed of the shocked gas should be larger than it is near the apex, resulting in larger emission line velocities. Since at those phases the shock 
cone points in our direction, the observed radial velocity should be shifted to very negative values, different to what is observed, unless the opening angle of the cone increases by a large factor.

In principle, the He II $\lambda 4686$ emission could be formed in the inner wind of the primary star, according to models computed by Hillier et al. (2001, 2006). The problem then would be to explain its disappearance at periastron, since at that phase the primary star is at our side.

Another possibility is that the stellar wind of the secondary star would be the source of the faint He II $\lambda 4686$ emission seen near apastron. Studies of the photoionization of the ejecta around the system suggest that the companion star has effective temperature between 37,000 and 43,000 $\mathrm{K}$ and luminosity between $10^{5}-10^{6} L_{\odot}$ (Mehner et al. 2010a; Verner et al. 2005; Teodoro et al. 2008). As discussed by Hillier et al. (2006), a companion star with $T_{\text {eff }}=33,000 \mathrm{~K}$ and $L=10^{6} L_{\odot}$ would not have its spectral lines detected in the optical, since the flux of the primary star is at least a factor of $\sim 25$ higher than that of the companion at those wavelengths.

In any case, if the secondary star was the source of the faint He II $\lambda 4686$ line observed far from periastron, the emission should be stronger near apastron than far from it, since the WWC cavity is opened toward us at those phases (Pittard \& Corcoran 2002; Okazaki et al. 2008; Parkin et al. 2009, 2011).

However, as seen in Fig. 4, the emission line intensity at $\phi=0.45$ (close to apastron) is not stronger than at $\phi=0.26$ or at $\phi=0.897$, when the secondary is closer to periastron. Therefore, the only region left to be responsible for the production of the He II $\lambda 4686$ at apastron is the WWC shock. In the next section, we will show that this line is formed in the WWC at periastron too.

\section{DISCUSSION}

Peak P2 (the absolute maximum) in the intrinsic luminosity of the He II $\lambda 4686$ line emission corresponds to an emitted power of $1.2 \times 10^{36} \mathrm{erg} \mathrm{s}^{-1}$ (recall that this is a lower limit because of uncertainties in the extinction correction). This large luminosity is emitted in only a single atomic transition. The secondary wind is already excluded as the source of the emission because the mechanism of illumination of its unshocked stellar wind by the hard UV/soft X-rays produced in the WWC region, proposed by Steiner \& Damineli (2004), does not account for the revised intrinsic photon flux of the He II $\lambda 4686$ line. There are only two regions in which this enormous He II $\lambda 4686$ luminosity could be produced: the shocked stellar wind of the primary or the shocked stellar wind of the secondary star.

Recent 3D models including inhibition effects (Parkin et al. 2009) show that the wind of the secondary star close to periastron must collide with a slower pre-shock velocity $\left(\sim 1,400 \mathrm{~km} \mathrm{~s}^{-1}\right)$ due to the strong radiation field of the primary star (a process known as radiative inhibition). In this case, the shocked wind of the secondary near periastron would produce emission at lower energies than if the winds collided at terminal velocity. This softer emission might lead to an increase of flux near the peak of the $\mathrm{He}^{+}$ionization threshold at $0.0544 \mathrm{keV}$.

To test this, we adopted the results of $3 \mathrm{D}$ simulations (Parkin et al. 2009, 2011) for the intrinsic spec- tral energy distribution (SED) of the shocked secondary's wind (Fig. 8) and used them to calculate the total power produced in the hard UV/soft X-ray domain during periastron passage (we extrapolated the SED down to $0.0544 \mathrm{keV}$ and then integrated it from 0.0544 to $0.550 \mathrm{keV})$. This model predicts an intrinsic luminosity $L^{0}=7 \times 10^{37} \mathrm{erg} \mathrm{s}^{-1}$ produced by the thermalization of the shocked secondary's wind. We then used the CLOUDY photoionization code to derive what fraction of the total He II recombination emission would correspond to the transition from $n=4$ to $n=3$ in a high excitation nebula with density of $n_{e} \sim 10^{10} \mathrm{~cm}^{-3}$ (suitable for the shocked secondary's wind). We found that He II $\lambda 4686$ accounts for less than 0.3 per cent of the total He II emission. Therefore, the secondary's thermalized shocked wind would have to have an intrinsic luminosity of

$$
L^{0}=\left(\frac{54.4}{4.2}\right)\left(\frac{L_{\lambda 4686}^{\mathrm{obs}}}{0.003}\right)=5.2 \times 10^{39} \mathrm{erg} \mathrm{s}^{-1}
$$

in order to account for the intrinsic luminosity of He II $\lambda 4686$. This value is almost 2 orders of magnitude higher than that calculated by 3D models, and therefore, we take this as strong evidence that the He II $\lambda 4686$ emission line does not originate in the secondary's shocked wind (even in the most favorable case where radiative inhibition causes the shock to occur at velocities lower than terminal).

The temperature, density and velocity of the primary's shocked stellar wind favor the production of photons with energy in the hard $\mathrm{UV} /$ soft X-ray domain suitable for ionizing or exciting $\mathrm{He}^{+}$. Inside the high density $\left(n_{e} \sim 10^{10} \mathrm{~cm}^{-3}\right)$ and low temperature $\left(<10^{5} \mathrm{~K}\right)$ shocked primary's wind, both the photoionization due to the X-ray and stellar radiation fields increase the extent of the $\mathrm{He}^{++}$region. This would naturally increase the number of recombining ions contributing to the line emission, which could account for the intrinsic luminosity of He II $\lambda 4686$ near periastron. Furthermore, radiative effects in $\mathrm{He}^{+}$(Martin et al. 2006) can also increase the population in the $n=2$ level from where $\mathrm{He}^{+}$can be easily ionized by any photon with $h \nu>13.6 \mathrm{eV}$ (i.e. photons in the hydrogen Lyman continuum). Therefore, the intrinsic SED of the shocked primary's wind from 3D models would be of great interest to determine the exact amount of He II $\lambda 4686$ emission within this region - this study, however, is beyond the scope of the present paper. Therefore, based on the intrinsically high luminosity of the He II $\lambda 4686$ emission line, we exclude all other possible regions but the primary's post-shocked wind as the region where the strong emission seen near periastron is formed.

The time delay of 16.5 days between the X-ray and the He II $\lambda 4686$ light curves could be determined by the time flow of the gas from the WWC apex up to the location where He II $\lambda 4686$ is emitted. The maximum distance could be estimated by adopting the flow velocity as the primary's wind terminal speed $\left(500 \mathrm{~km} \mathrm{~s}^{-1}\right)$, which amounts to $4-5 \mathrm{AU}$ from the apex. However, the real distance should be much smaller than that, since close to the apex the winds collide almost radially. The high density at periastron leads to very fast cooling, so that the He II $\lambda 4686$ line should be formed not very far from 


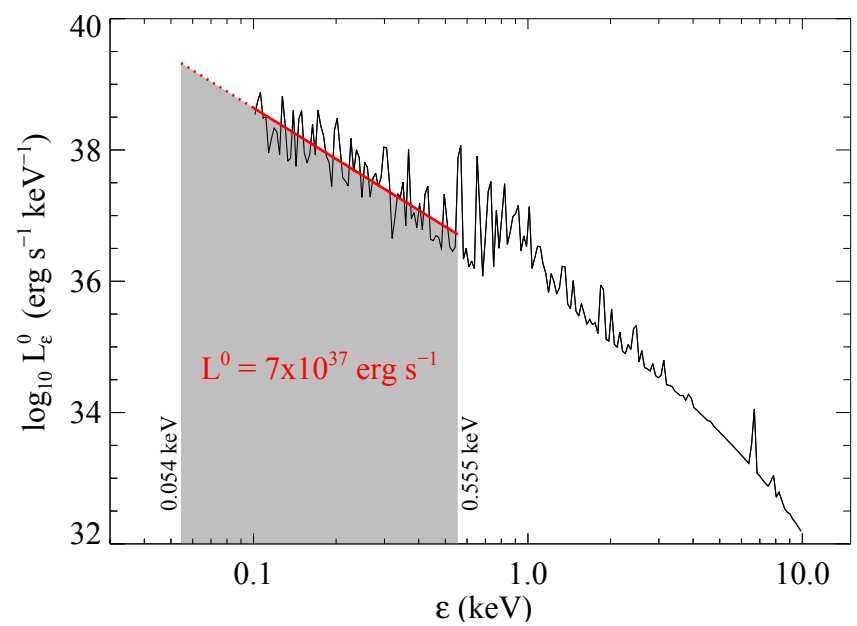

Figure 8. Calculated spectral energy distribution (SED) of the shocked secondary's wind during periastron passage (from Parkin et al. 2009). The intrinsic luminosity, $L^{0}$, was obtained by integrating the SED from 0.0544 to $0.550 \mathrm{keV}$ (indicated by the gray shaded area). Notice that we fitted the SED (solid red line) and extrapolated it in the range from 0.0544 to $0.1 \mathrm{keV}$ (dotted red line) because the output from simulations only goes down to $0.1 \mathrm{keV}$.

the WWC apex. Using the Hillier et al. (2001) model, we derive impact parameter of the primary's wind in the broad band $B$-filter (which encompasses the He II $\lambda 4686$ line) as $\mathrm{D}_{B}=4.4 \mathrm{AU}$. Since the He II $\lambda 4686$ emitting region is close to the WWC apex, which is very close to the primary star at periastron it could be eclipsed by the wind of the primary star for an intermediate orbital inclination. The expected duration of the eclipse of the He II $\lambda 4686$ emitting region by the primary's stellar wind would be shorter than the X-ray emitting region, as the former is larger than the latter. In fact, the 2009.0 minimum in He II $\lambda 4686$ lasted for a week as compared to the 4 weeks for the X-ray minimum. Both minima were centered at $\phi=0.005$, reinforcing the nature of the He II $\lambda 4686$ and the X-ray minima as due to occultation.

Soon after the minimum, the He II $\lambda 4686$ starts rising again, reaching a local maximum (P3) in the phase range $\Delta \phi=0.01-0.03$ (a duration of 40 days). Interestingly, the $2-10 \mathrm{keV}$ X-ray emission increased very slowly at that time interval. We interpret this as a collapse of the WWC shock into deeper regions of the secondary stellar wind, where it is still being accelerated. As discussed by Parkin et al. (2009), Corcoran et al. (2010) and Parkin et al. (2011), radiative inhibition of the secondary's wind might lead to a collapse of the WWC onto the "surface" of the secondary when stars approach periastron. Of course, since the secondary star has a strong wind, it is likely that the momentum balance occurs at a radius larger than its photosphere. Accretion onto the companion star might also play a role (Soker \& Behar 2006; Soker 2007), but is not a necessary condition to describe the observed phenomena. Thus, the secondary's wind is dominated by the radiation from the primary star (radiative inhibition) for a brief period and the hard X-ray source near the WWC apex is switched off. The associated He II $\lambda 4686$ emission may be absent during this time until the WWC forms anew. In the collapsed state, the WWC shock cone should be narrower, but still a powerful source at extreme UV energies, since the stellar winds continue to collide downstream of the stagnation

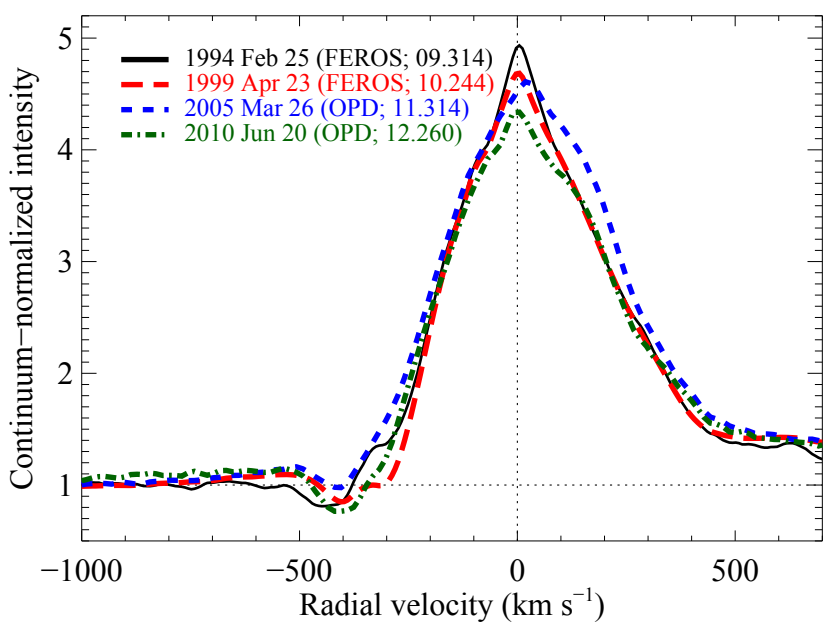

Figure 9. Continuum-normalized line profile of $\mathrm{H} \delta$ at approximately the same phase (indicated in the legend), along the last four cycles. Moderate variability at a low level is present, but no strong change in the line strength (which would be expected if the mass loss rate dropped by a factor of 2) was seen in any of the four cycles.

point.

As the stars separate after periastron passage, there should be a point in the orbit when the winds collide at their full terminal speeds once again. At that time, the WWC shock would be restored, and consequently the hard X-rays emission would restart. However, the wind of the secondary needs to do extra work to push back the primary's wind. This is probably not a smooth process, because the momentum equilibrium in the WWC apex does not only depend on the global properties of the winds, but also on local properties: clumps driven by radiative instabilities in the primary wind (Moffat \& Corcoran 2009) might produce stochastic variability as the wind-wind shock front tries to reform. Changes in the clump properties could in principle explain why the minimum in X-rays was much shorter in cycle \#12 than in cycles \#11 and \#10, without needing to invoke a decrease by a factor of two in the mass-loss rate of the primary star (Corcoran et al. 2010). Also, lines formed deep in the primary's wind do not show particular changes in cycle \#12 compared to earlier cycles. Fig. 9 displays line profiles of the line $\mathrm{H} \delta$ taken almost at the same orbital phase $(\phi \sim 0.3)$ along the last 4 cycles (data obtained with FEROS/ESO and OPD/LNA). There are only small-scale variations in the $\mathrm{P}$ Cygni absorption strength and terminal speed. This line is relevant, since it is formed in regions of the primary's wind little affected by the WWC shock in the interval $\Delta \phi=0.2-0.8$, as can be seen in Fig. 10 (which is based on the model by Hillier et al. 2001). In this figure, the circles indicate the radii of maximum emissivity for different spectral lines. We also indicate the zone inside which the $\mathrm{H} \delta$ emissivity is larger than 50 per cent of the peak intensity (dotted circle). $\mathrm{H} \alpha$ is affected by the WWC for most of the orbit and Fe II lines, formed at much larger distances from the central star ${ }^{11}$, are disturbed by the WWC even at apastron. In this way, changes reported by Mehner et al.

11 For example, the Fe II $\lambda 4923$ emissivity is larger than 50 per cent of the peak emissivity within a ring which goes from $\sim 25$ to 140 A.U. from the primary star. 


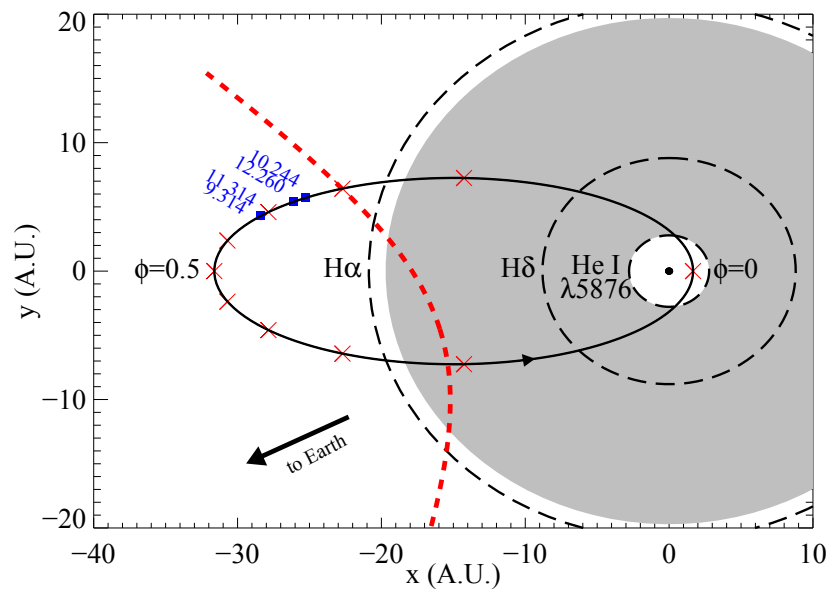

Figure 10. Projection of the orbital plane, relative to the position of the primary star ( $e=0.9$ and $P=2022.7$ days). The concentric dashed circles correspond to the region of maximum emissivity of each labelled line. The gray shaded area indicates the zone where $\mathrm{H} \delta$ emissivity is higher than 50 per cent of its maximum. The primary star is drawn to scale, with $R_{*}=60 R_{\odot}$. The red $\times$ symbols are drawn at each 0.1 in phase and the blue squares and numbers indicate the cycle+phase when the spectra shown in Fig. 9 were obtained. The dashed paraboloid indicates the position of the WWC shock at $\phi=0.8$ and stellar mass of $90 M_{\odot}$ and $30 M_{\odot}$ for the primary and secondary star, respectively.

(2010b) in those lines cannot be attributed to the global properties of the primary's wind, but are probably driven by fluctuations in the WWC.

\section{CONCLUSIONS}

In this paper, we present data from the last 4 low excitation events in $\eta$ Car in the light of the He II $\lambda 4686$ emission line. We summarize our results and conclusions below.

- The He II $\lambda 4686$ line is present at a faint level along most of the 5.538 yr cycle;

- two months before periastron, the He II $\lambda 4686$ line strength increases dramatically with large flare-like oscillations on a timescale of weeks, similar to the X-ray "flares" seen just prior to periastron passage;

- in the 2009.0 event, just before the minimum, there were 2 distinct peaks (P1 and P2) in the line equivalent width. Peaks $\mathrm{P} 1$ and $\mathrm{P} 2$ are correlated with flares seen in the X-rays light curve, after shifting the later by +16.5 days. They are not phase-locked and are probably due to localized density enhancements (clumps) in the primary's stellar wind;

- there is an abrupt decrease in the line emission, reaching a minimum centered at $\phi=0.005$. This minimum is centered at the same phase as the Xray minimum, but lasts only a week. We argue that this minimum is an occultation of the He II $\lambda 4686$ emitting region by the primary's stellar wind;

- the fact that the disappearance of He II $\lambda 4686$ emission in the central source and at the FOS4 position (in the SE lobe of the Homunculus) occurs close in time (but not simultaneously) is not an argument against an occultation, since the binary system revolves very quickly at periastron;
- in the 2009.0 event, after the minimum, the He II $\lambda 4686$ equivalent width shows another peak (P3), which appears to repeat from cycle to cycle. This peak is not correlated with the X-ray variation, as the He II $\lambda 4686$ emission is in the high state when X-rays are very low. This He II $\lambda 4686$ peak occurs in the phase range $\Delta \phi=1.01-1.035$;

- a potential mechanism to explain the P3 peak is a collapse of the WWC onto the "surface" of the secondary star due to radiative inhibition of the secondary's wind by photons from the primary star, which could shift most of the energy of the shocked gas to lower energies near the $\mathrm{He}^{+}$ionization threshold;

- if the collapse scenario is valid, periastron occurs at $\phi \sim 0.02$ or a little earlier;

- the He II $\lambda 4686$ equivalent width did not display the early recovery seen in X-rays after the 2009.0 event. We suggest that the recovery of the WWC region from the collapse phase is driven by localized instabilities/clumps in the primary's wind as suggested by Akashi et al. (2006). Moffat \& Corcoran (2009) suggested that clumps might explain flares in the X-ray light-curve, and might control the momentum balance at scales on the clump size $(\sim 1 \mathrm{AU})$.

We thank the referee for questions and suggestions that led to a clarification of the ideas discussed in this paper. MT, AD and JES are grateful to the Brazilian agencies FAPESP and CNPq for continuous financial support. MT is supported through grants FAPESP 05/00190-8 and 09/08013-9. MBF acknowledges Conselho Nacional de Desenvolvimento Científico e Tecnológico (CNPqBrazil) for the post-doctoral grant. MFC gratefully acknowledges support from NASA and Chandra via grants G07-8022A, G08-9018A, G09-0016A, and GO0-11039A, along with continued aid from the RXTE Guest Observer facility at NASA/GSFC. Calculations were performed with version 07.02 of Cloudy, last described by Ferland et al. (1998). This research has made use of NASA's Astrophysics Data System. In addition, this research has made use of data obtained from the High Energy Astrophysics Science Archive Research Center (HEASARC), provided by NASA's Goddard Space Flight Center.

Facilities: SOAR: 4.1m (Goodman), CASLEO: $2.15 \mathrm{~m}$ (REOSC and EBASIM), ESO: 2.2m (FEROS), LNA: 1.6m (Coudé), Magellan: 6.5m Clay (MIKE), MtS: $1.9 \mathrm{~m}$ (Coudé) .

\section{REFERENCES}

Abraham, Z. \& Falceta-Gonçalves, D. 2007, Monthly Notices of the Royal Astronomical Society, 378, 309

Abraham, Z., Falceta-Gonçalves, D., Dominici, T., Caproni, A., \& Jatenco-Pereira, V. 2005, Monthly Notices of the Royal Astronomical Society, 364, 922

Akashi, M., Soker, N., \& Behar, E. 2006, The Astrophysical Journal, 644, 451

Bartzakos, P., Moffat, A. F. J., \& Niemela, V. S. 2001, Monthly Notices of the Royal Astronomical Society, 324, 33 
Breysacher, J. \& François, P. 2000, Astronomy and Astrophysics, 361,231

Cardelli, J. A., Clayton, G. C., \& Mathis, J. S. 1989, ApJ, 345, 245

Corcoran, M. F. 2005, The Astronomical Journal, 129, 2018

Corcoran, M. F., Hamaguchi, K., Pittard, J. M., Russell, C. M. P., Owocki, S. P., Parkin, E. R., \& Okazaki, A. 2010, The Astrophysical Journal, 725, 1528

Corcoran, M. F., Pollock, A. M. T., Hamaguchi, K., \& Russell, C. 2011, eprint arXiv:1101.1422

Damineli, A. 1996, Astrophysical Journal Letters v.460, 460, L49 - 1997, Luminous Blue Variables: Massive Stars in Transition. ASP Conference Series; Vol. 120; 1997; ed. Antonella Nota and Henny Lamers (1997), 120, 272

Damineli, A., Conti, P. S., \& Lopes, D. F. 1997, New Astronomy, 2,107

Damineli, A., Hillier, D. J., Corcoran, M. F., Stahl, O., Groh, J. H., Arias, J., Teodoro, M., Morrell, N., Gamen, R., Gonzalez, F., Leister, N. V., Levato, H., Levenhagen, R. S., Grosso, M., Colombo, J. F. A., \& Wallerstein, G. 2008a, Monthly Notices of the Royal Astronomical Society, 386, 2330

Damineli, A., Hillier, D. J., Corcoran, M. F., Stahl, O., Levenhagen, R. S., Leister, N. V., Groh, J. H., Teodoro, M., Albacete Colombo, J. F., Gonzalez, F., Arias, J., Levato, H., Grosso, M., Morrell, N., Gamen, R., Wallerstein, G., \& Niemela, V. 2008b, Monthly Notices of the Royal Astronomical Society, 384, 1649

Damineli, A., Kaufer, A., Wolf, B., Stahl, O., Lopes, D. F., \& de Araújo, F. X. 2000, The Astrophysical Journal, 528, L101

Damineli, A., Lopes, D. F., \& Conti, P. S. 1999, Eta Carinae At The Millennium, 179, 288

Damineli, A., Stahl, O., Kaufer, A., Wolf, B., Quast, G., \& Lopes, D. F. 1998, Astronomy and Astrophysics Supplement, 133, 299

Davidson, K., Ebbets, D., Johansson, S., Morse, J. A., \&

Hamann, F. W. 1997, Astronomical Journal v.113, 113, 335

Davidson, K., Ebbets, D., Weigelt, G., Humphreys, R. M., Hajian, A. R., Walborn, N. R., \& Rosa, M. 1995, The Astronomical Journal, 109, 1784

Davidson, K., Martin, J., Humphreys, R. M., Ishibashi, K., Gull, T. R., Stahl, O., Weis, K., Hillier, D. J., Damineli, A., Corcoran, M., \& Hamann, F. 2005, The Astronomical Journal, 129,900

de la Chevrotière, A., Moffat, A. F. J., \& Chené, A. N. 2011, Monthly Notices of the Royal Astronomical Society, 411, 635

Dorland, B. N., Currie, D. G., \& Hajian, A. R. 2004, AJ, 127, 1052

Fahed, R., Moffat, A. F. J., Zorec, J., Eversberg, T., Chené, A. N., Alves, F., Arnold, W., Bergmann, T., Gouveia Carreira, L. F., Marques Dias, F., Fernando, A., Sanchez Gallego, J., Hunger, T., Knapen, J., Leadbeater, R., Morel, T., Rauw, G., Reinecke, N., Ribeiro, J., Romeo, N., dos Santos, E. M., Schanne, L., Stahl, O., Stober, B., Stober, B., Correia Viegas, N. G., Vollmann, K., Corcoran, M. F., Dougherty, S. M., Pittard, J. M., Pollock, A. M. T., \& Williams, P. M. 2011, Société Royale des Sciences de Liège, 80, 668

Falceta-Gonçalves, D. \& Abraham, Z. 2009, MNRAS, 1245

Ferland, G. J., Korista, K. T., Verner, D. A., Ferguson, J. W., Kingdon, J. B., \& Verner, E. M. 1998, PASP, 110, 761

Fernandez Lajus, E., Farina, C., Calderon, J. P., Salerno, N., Torres, A. F., Schwartz, M. A., von Essen, C., Giudici, F., \& Bareilles, F. A. 2010, New Astronomy, 15, 108

Fernandez Lajus, E., Farina, C., Torres, A. F., Schwartz, M. A., Salerno, N., Calderon, J. P., von Essen, C., Calcaferro, L. M. Giudici, F., Llinares, C., \& Niemela, V. 2009, Astronomy and Astrophysics, 493, 1093

Flores, A., Auer, L. H., Koenigsberger, G., \& Cardona, O. 2001, The Astrophysical Journal, 563, 341

Gaviola, E. 1953, ApJ, 118, 234

Groh, J. H. \& Damineli, A. 2004, Information Bulletin on Variable Stars, 5492, 1

Groh, J. H., Nielsen, K. E., Damineli, A., Gull, T. R., Madura, T. I., Hillier, D. J., Teodoro, M., Driebe, T., Weigelt, G., Hartman, H., Kerber, F., Okazaki, A. T., Owocki, S. P., Millour, F., Murakawa, K., Kraus, S., Hofmann, K.-H., \& Schertl, D. 2010, Astronomy and Astrophysics, 517, 9

Gull, T. R., Madura, T. I., Groh, J. H., \& Corcoran, M. F. 2011, eprint arXiv: 1110.6420
Gull, T. R., Nielsen, K. E., Corcoran, M. F., Madura, T. I., Owocki, S. P., Russell, C. M. P., Hillier, D. J., Hamaguchi, K., Kober, G. V., Weis, K., Stahl, O., \& Okazaki, A. T. 2009, MNRAS, 396, 1308

Hamaguchi, K., Corcoran, M. F., Gull, T., Ishibashi, K., Pittard, J. M., Hillier, D. J., Damineli, A., Davidson, K., Nielsen, K. E., \& Kober, G. V. 2007, ApJ, 663, 522

Henley, D. B., Corcoran, M. F., Pittard, J. M., Stevens, I. R., Hamaguchi, K., \& Gull, T. R. 2008, The Astrophysical Journal, 680,705

Hill, G. M., Moffat, A. F. J., \& St-Louis, N. 2002, Monthly Notices of the Royal Astronomical Society, 335, 1069

Hillier, D. J., Davidson, K., Ishibashi, K., \& Gull, T. R. 2001, The Astrophysical Journal, 553, 837

Hillier, D. J., Gull, T., Nielsen, K., Sonneborn, G., Iping, R., Smith, N., Corcoran, M., Damineli, A., Hamann, F. W., Martin, J. C., \& Weis, K. 2006, The Astrophysical Journal, 642,1098

Hubrig, S., Briquet, M., Morel, T., Schöller, M., González, J. F., \& de Cat, P. 2008, Astronomy and Astrophysics, 488, 287

Ishibashi, K., Corcoran, M. F., Davidson, K., Swank, J. H., Petre, R., Drake, S. A., Damineli, A., \& White, S. 1999, The Astrophysical Journal, 524, 983

Kashi, A. \& Soker, N. 2008, Monthly Notices of the Royal Astronomical Society, 390, 1751

Kaufer, A., Stahl, O., Tubbesing, S., Nørregaard, P., Avila, G., Francois, P., Pasquini, L., \& Pizzella, A. 1999, The Messenger, 95,8

Koenigsberger, G., Georgiev, L., Hillier, D. J., Morrell, N., Barbá, R., \& Gamen, R. 2010, The Astronomical Journal, 139, 2600

Madura, T. I., Gull, T. R., Owocki, S. P., Groh, J. H., Okazaki, A. T., \& Russell, C. M. P. 2011, eprint arXiv:1111.2226

Marchenko, S. V., Moffat, A. F. J., Ballereau, D., Chauville, J., Zorec, J., Hill, G. M., Annuk, K., Corral, L. J., Demers, H., Eenens, P. R. J., Panov, K. P., Seggewiss, W., Thomson, J. R., \& Villar Sbaffi, A. 2003, ApJ, 596, 1295

Marchenko, S. V., Moffat, A. F. J., Eenens, P. R. J., Cardona, O., Echevarria, J., \& Hervieux, Y. 1997, Astrophysical Journal v. $485,485,826$

Martin, J. C., Davidson, K., Humphreys, R. M., Hillier, D. J., \& Ishibashi, K. 2006, The Astrophysical Journal, 640, 474

McGregor, P. J., Rathborne, J. M., \& Humphreys, R. M. 1999, Eta Carinae At The Millennium, 179, 236

Mehner, A., Davidson, K., Ferland, G. J., \& Humphreys, R. M. 2010a, The Astrophysical Journal, 710, 729

Mehner, A., Davidson, K., Humphreys, R. M., Martin, J. C., Ishibashi, K., Ferland, G. J., \& Walborn, N. R. 2010b, The Astrophysical Journal Letters, 717, L22

Moffat, A. F. J. \& Corcoran, M. F. 2009, The Astrophysical Journal, 707, 693

Moffat, A. F. J., Marchenko, S. V., Bartzakos, P., Niemela, V. S., Cerruti, M. A., Magalhaes, A. M., Balona, L., St-Louis, N., Seggewiss, W., \& Lamontagne, R. 1998, ApJ, 497, 896

Nazé, Y. \& Rauw, G. 2008, Astronomy and Astrophysics, 490, 801

Okazaki, A. T., Owocki, S. P., Russell, C. M. P., \& Corcoran, M. F. 2008, Monthly Notices of the Royal Astronomical Society: Letters, 388, L39

Paczynski, B. 1998, ApJS, 494, L45

Parkin, E. R., Pittard, J. M., Corcoran, M. F., \& Hamaguchi, K. 2011, The Astrophysical Journal, 726, 105

Parkin, E. R., Pittard, J. M., Corcoran, M. F., Hamaguchi, K., \& Stevens, I. R. 2009, Monthly Notices of the Royal Astronomical Society, 394, 1758

Pittard, J. M. \& Corcoran, M. F. 2002, Astronomy and Astrophysics, 383, 636

Rodgers, A. W. \& Searle, L. 1967, Monthly Notices of the Royal Astronomical Society, 135, 99

Schnurr, O., Moffat, A. F. J., Villar Sbaffi, A., St-Louis, N., \& Morrell, N. I. 2009, Monthly Notices of the Royal Astronomical Society, 395, 823

Smith, N. \& Frew, D. J. 2011, Monthly Notices of the Royal Astronomical Society, 415, 2009

Smith, N., Li, W., Foley, R. J., Wheeler, J. C., Pooley, D., Chornock, R., Filippenko, A. V., Silverman, J. M., Quimby, R., Bloom, J. S., \& Hansen, C. 2007, The Astrophysical Journal, 666, 1116 
Smith, N., Morse, J. A., Gull, T. R., Hillier, D. J., Gehrz, R. D., Walborn, N. R., Bautista, M., Collins, N. R., Corcoran, M. F., Damineli, A., Hamann, F., Hartman, H., Johansson, S., Stahl, O., \& Weis, K. 2004, The Astrophysical Journal, 605, 405

Soker, N. 2003, ApJ, 597, 513

-. 2005, The Astrophysical Journal, 635, 540

—. 2007, The Astrophysical Journal, 661, 482

Soker, N. \& Behar, E. 2006, The Astrophysical Journal, 652, 1563

Sota, A., Maíz Apellániz, J., Walborn, N. R., Alfaro, E. J.

Barbá, R. H., Morrell, N. I., Gamen, R. C., \& Arias, J. I. 2011, The Astrophysical Journal Supplement, 193, 24

Steiner, J. E. \& Damineli, A. 2004, The Astrophysical Journal, 612, L133

Teodoro, M., Damineli, A., Sharp, R. G., Groh, J. H., \& Barbosa, C. L. 2008, Monthly Notices of the Royal Astronomical Society, 387,564

Thackeray, A. D. 1953, Monthly Notices of the Royal Astronomical Society, 113, 211

-. 1967, Monthly Notices of the Royal Astronomical Society, 135,51

Tody, D. 1993, Astronomical Data Analysis Software and Systems II, 52,173

Usov, V. V. 1992, ApJS, 389, 635 van Genderen, A. M., Sterken, C., Allen, W. H., \& Walker, W.

S. G. 2006, Journal of Astronomical Data, 12, 3

Verner, E., Bruhweiler, F., \& Gull, T. 2005, ApJ, 624, 973

Walborn, N. R., Sota, A., Maíz Apellániz, J., Alfaro, E. J.

Morrell, N. I., Barba, R. H., Arias, J. I., \& Gamen, R. C. 2010, ApJS, 711, L143

Weigelt, G., Albrecht, R., Barbieri, C., Blades, J. C., Boksenberg, A., Crane, P., Davidson, K., Deharveng, J. M., Disney, M. J., Jakobsen, P., Kamperman, T. M., King, I. R., Macchetto, F., Mackay, C. D., Paresce, F., Baxter, D., Greenfield, P., Jedrzejewski, R., Nota, A., \& Sparks, W. B. 1995, Revista

Mexicana de Astronomia y Astrofisica Serie de Conferencias, 2, 11

Weigelt, G. \& Ebersberger, J. 1986, A\&A, 163, L5

White, R. L. \& Becker, R. H. 1995, ApJ, 451, 352

Whitelock, P. A., Feast, M. W., Koen, C., Roberts, G., \& Carter, B. S. 1994, R.A.S. MONTHLY NOTICES V.270, 270, 364

Williams, P. 2011, Bulletin de la Societe Royale des Sciences de Liege, 80, 595

Williams, P. M., van der Hucht, K. A., Pollock, A. M. T.,

Florkowski, D. R., van der Woerd, H., \& Wamsteker, W. M. 1990, Monthly Notices of the Royal Astronomical Society, 243, 662

Zanella, R., Wolf, B., \& Stahl, O. 1984, A\&A, 137, 79 
Table 1

Journal of observations and measurements for the low-excitation events \#9, \#10, \#11 and \#12.

\begin{tabular}{|c|c|c|c|c|}
\hline JD & $\begin{array}{c}\text { cycle } \\
+ \\
\text { phase }\end{array}$ & $\begin{array}{l}\text { Equivalent } \\
\text { width } \\
(\AA)\end{array}$ & $\begin{array}{l}\text { Radial velocity } \\
\text { of the peak } \\
\left(\mathrm{km} \mathrm{s}^{-1}\right)\end{array}$ & $\begin{array}{c}\text { Observatory } \\
+ \\
\text { Instrument }\end{array}$ \\
\hline 2448776.4 & 9.001 & $-1.27 \pm 0.10$ & -293 & OPD+Coudé \\
\hline 2448794.5 & 9.010 & $-0.31 \pm 0.10$ & -75 & ESO/La Silla+FLASH/HEROS \\
\hline 2448825.5 & 9.025 & $-0.56 \pm 0.10$ & -25 & ESO/La Silla+FLASH/HEROS \\
\hline 2448830.5 & 9.028 & $-0.36 \pm 0.10$ & -10 & $\mathrm{ESO} / \mathrm{La}$ Silla+FLASH/HEROS \\
\hline 2448839.5 & 9.032 & $-0.26 \pm 0.10$ & -40 & ESO/La Silla+FLASH/HEROS \\
\hline 2448844.5 & 9.035 & $-0.19 \pm 0.10$ & -35 & ESO/La Silla+FLASH/HEROS \\
\hline 2450771.1 & 9.987 & $-1.93 \pm 0.10$ & -240 & MSSSO+Coudé \\
\hline 2450808.2 & 10.006 & $0.20 \pm 0.10$ & N.M. & MSSSO+Coudé \\
\hline 2450869.9 & 10.036 & $-0.19 \pm 0.10$ & -34 & MSSSO+Coudé \\
\hline 2450896.1 & 10.049 & $-0.15 \pm 0.10$ & -10 & MSSSO+Coudé \\
\hline 2452773.5 & 10.977 & $-0.72 \pm 0.12$ & -45 & OPD+Coudé \\
\hline 2452803.5 & 10.992 & $-1.48 \pm 0.10$ & -248 & OPD+Coudé \\
\hline 2452811.4 & 10.996 & $-2.21 \pm 0.55$ & -266 & OPD+Coudé \\
\hline 2452812.4 & 10.996 & $-2.67 \pm 0.10$ & -264 & OPD+Coudé \\
\hline 2452813.5 & 10.997 & $-2.30 \pm 0.12$ & -295 & OPD+Coudé \\
\hline 2452814.4 & 10.997 & $-2.36 \pm 0.63$ & -349 & OPD+Coudé \\
\hline 2452815.4 & 10.998 & $-2.80 \pm 0.14$ & -356 & OPD+Coudé \\
\hline 2452816.4 & 10.998 & $-2.88 \pm 0.13$ & -356 & OPD+Coudé \\
\hline 2452817.4 & 10.999 & $-2.48 \pm 0.10$ & -368 & OPD+Coudé \\
\hline 2452818.4 & 10.999 & $-1.93 \pm 0.10$ & -390 & OPD+Coudé \\
\hline 2452819.4 & 11.000 & $-1.75 \pm 0.14$ & -405 & OPD+Coudé \\
\hline 2452820.5 & 11.000 & $-1.16 \pm 0.10$ & -432 & OPD+Coudé \\
\hline 2452821.4 & 11.001 & $-0.48 \pm 0.39$ & -442 & OPD+Coudé \\
\hline 2452822.4 & 11.001 & $-0.29 \pm 0.11$ & -402 & OPD+Coudé \\
\hline 2452823.4 & 11.002 & $-0.09 \pm 0.11$ & -460 & OPD+Coudé \\
\hline 2452825.5 & 11.003 & $0.00 \pm 0.10$ & N.M. & OPD+Coudé \\
\hline 2452826.4 & 11.003 & $-0.08 \pm 0.10$ & N.M. & OPD+Coudé \\
\hline 2452827.4 & 11.004 & $-0.08 \pm 0.10$ & N.M. & OPD+Coudé \\
\hline 2452828.4 & 11.004 & $-0.07 \pm 0.10$ & N.M. & OPD+Coudé \\
\hline 2452830.5 & 11.005 & $-0.09 \pm 0.10$ & N.M. & OPD+Coudé \\
\hline 2452841.4 & 11.011 & $-0.65 \pm 0.16$ & -24 & OPD+Coudé \\
\hline 2452987.7 & 11.083 & $-0.12 \pm 0.17$ & -1 & OPD+Coudé \\
\hline 2454190.7 & 11.678 & $-0.04 \pm 0.12$ & -97 & OPD+Coudé \\
\hline 2454311.4 & 11.737 & $-0.07 \pm 0.13$ & -1 & OPD+Coudé \\
\hline 2454595.4 & 11.878 & $-0.07 \pm 0.12$ & +5 & OPD+Coudé \\
\hline 2454596.5 & 11.878 & $-0.11 \pm 0.10$ & +4 & OPD+Coudé \\
\hline 2454598.4 & 11.879 & $-0.08 \pm 0.10$ & -4 & OPD+Coudé \\
\hline 2454774.5 & 11.966 & $-0.55 \pm 0.20$ & -50 & $\mathrm{LCO}+\mathrm{MIKE}$ \\
\hline 2454774.8 & 11.967 & $-0.55 \pm 0.20$ & -50 & $\mathrm{LCO}+\mathrm{MIKE}$ \\
\hline 2454784.9 & 11.972 & $-0.43 \pm 0.20$ & -75 & $\mathrm{LCO}+\mathrm{MIKE}$ \\
\hline 2454786.0 & 11.972 & $-0.42 \pm 0.05$ & -70 & CASLEO+EBASIM \\
\hline 2454787.0 & 11.973 & $-0.34 \pm 0.01$ & -75 & CASLEO+EBASIM \\
\hline 2454803.8 & 11.981 & $-0.61 \pm 0.16$ & -101 & SOAR+Goodman \\
\hline 2454809.8 & 11.984 & $-1.32 \pm 0.14$ & -109 & SOAR+Goodman \\
\hline 2454815.8 & 11.987 & $-2.19 \pm 0.20$ & -127 & $\mathrm{LCO}+\mathrm{MIKE}$ \\
\hline 2454815.8 & 11.987 & $-2.30 \pm 0.11$ & -154 & SOAR+Goodman \\
\hline 2454821.7 & 11.990 & $-1.71 \pm 0.12$ & -175 & SOAR+Goodman \\
\hline 2454821.8 & 11.990 & $-1.74 \pm 0.11$ & -155 & ESO/La Silla+FEROS \\
\hline 2454822.9 & 11.990 & $-1.81 \pm 0.10$ & -153 & ESO/La Silla+FEROS \\
\hline 2454823.8 & 11.991 & $-1.78 \pm 0.11$ & -151 & ESO/La Silla+FEROS \\
\hline 2454824.8 & 11.991 & $-1.69 \pm 0.11$ & -163 & ESO/La Silla+FEROS \\
\hline 2454825.8 & 11.992 & $-1.75 \pm 0.11$ & -156 & ESO/La Silla+FEROS \\
\hline 2454826.8 & 11.992 & $-1.65 \pm 0.11$ & -146 & SOAR+Goodman \\
\hline 2454827.8 & 11.993 & $-1.70 \pm 0.14$ & -195 & SOAR+Goodman \\
\hline 2454827.9 & 11.993 & $-1.61 \pm 0.11$ & -163 & ESO/La Silla+FEROS \\
\hline 2454828.8 & 11.993 & $-1.53 \pm 0.10$ & -166 & SOAR+Goodman \\
\hline 2454828.9 & 11.993 & $-1.49 \pm 0.12$ & -168 & ESO/La Silla+FEROS \\
\hline 2454829.8 & 11.994 & $-1.45 \pm 0.11$ & -187 & SOAR+Goodman \\
\hline 2454829.9 & 11.994 & $-1.40 \pm 0.11$ & -196 & ESO/La Silla+FEROS \\
\hline 2454830.8 & 11.994 & $-1.47 \pm 0.10$ & -260 & SOAR+Goodman \\
\hline 2454830.9 & 11.994 & $-1.46 \pm 0.10$ & -228 & ESO/La Silla+FEROS \\
\hline 2454831.8 & 11.995 & $-1.87 \pm 0.11$ & -255 & SOAAR+Goodman \\
\hline 2454831.9 & 11.995 & $-1.85 \pm 0.12$ & -250 & ESO/La Silla+FEROS \\
\hline 2454832.9 & 11.995 & $-2.55 \pm 0.11$ & -224 & ESO/La Silla+FEROS \\
\hline 2454833.9 & 11.996 & $-2.77 \pm 0.11$ & -218 & ESO/La Silla+FEROS \\
\hline 2454834.8 & 11.996 & $-2.48 \pm 0.11$ & -269 & SOAR+Goodman \\
\hline 2454834.9 & 11.996 & $-2.54 \pm 0.11$ & -248 & ESO/La Silla+FEROS \\
\hline 2454835.0 & 11.996 & $-2.34 \pm 0.01$ & -314 & CASLEO+REOSC \\
\hline 2454835.8 & 11.997 & $-2.40 \pm 0.10$ & -319 & SOAR+Goodman \\
\hline 2454836.0 & 11.997 & $-2.49 \pm 0.01$ & -378 & CASLEO+REOSC \\
\hline 2454837.0 & 11.997 & $-2.40 \pm 0.01$ & -362 & CASLEO+REOSC \\
\hline 2454837.8 & 11.998 & $-2.57 \pm 0.13$ & -341 & SOAR+Goodman \\
\hline
\end{tabular}


Table 1 - Continued

\begin{tabular}{|c|c|c|c|c|}
\hline JD & $\begin{array}{c}\text { cycle } \\
+ \\
\text { phase }\end{array}$ & $\begin{array}{l}\text { Equivalent } \\
\text { width } \\
(\AA)\end{array}$ & $\begin{array}{l}\text { Radial velocity } \\
\text { of the peak } \\
\left(\mathrm{km} \mathrm{s}^{-1}\right)\end{array}$ & $\begin{array}{c}\text { Observatory } \\
+ \\
\text { Instrument }\end{array}$ \\
\hline 2454838.0 & 11.998 & $-2.08 \pm 0.01$ & -336 & CASLEO+REOSC \\
\hline 2454839.8 & 11.999 & $-1.90 \pm 0.13$ & -340 & SOAR+Goodman \\
\hline 2454840.0 & 11.999 & $-1.91 \pm 0.01$ & -349 & CASLEO+REOSC \\
\hline 2454841.0 & 11.999 & $-1.79 \pm 0.01$ & -370 & CASLEO+REOSC \\
\hline 2454841.8 & 12.000 & $-1.67 \pm 0.12$ & -362 & SOAR+Goodman \\
\hline 2454842.0 & 12.000 & $-1.87 \pm 0.01$ & -383 & CASLEO+REOSC \\
\hline 2454842.8 & 12.000 & $-1.48 \pm 0.10$ & -389 & SOAR+Goodman \\
\hline 2454843.7 & 12.001 & $-1.21 \pm 0.12$ & -412 & OPD+Coudé \\
\hline 2454843.8 & 12.001 & $-0.93 \pm 0.10$ & -420 & SOAR+Goodman \\
\hline 2454844.0 & 12.001 & $-0.65 \pm 0.01$ & -427 & CASLEO+REOSC \\
\hline 2454844.7 & 12.001 & $-0.89 \pm 0.11$ & -387 & OPD+Coudé \\
\hline 2454844.8 & 12.001 & $-0.63 \pm 0.11$ & -424 & SOAR+Goodman \\
\hline 2454845.0 & 12.001 & $-0.63 \pm 0.01$ & -452 & CASLEO+REOSC \\
\hline 2454845.8 & 12.002 & $-0.32 \pm 0.10$ & -393 & SOAR+Goodman \\
\hline 2454846.8 & 12.002 & $-0.09 \pm 0.11$ & -411 & SOAR+Goodman \\
\hline 2454847.0 & 12.002 & $-0.04 \pm 0.01$ & -425 & CASLEO+REOSC \\
\hline 2454847.8 & 12.003 & $0.04 \pm 0.10$ & N.M. & SOAR+Goodman \\
\hline 2454848.0 & 12.003 & $0.02 \pm 0.01$ & N.M. & CASLEO+REOSC \\
\hline 2454848.8 & 12.003 & $-0.00 \pm 0.20$ & N.M. & $\mathrm{LCO}+\mathrm{MIKE}$ \\
\hline 2454849.0 & 12.003 & $-0.09 \pm 0.01$ & N.M. & CASLEO+REOSC \\
\hline 2454850.0 & 12.004 & $0.08 \pm 0.01$ & N.M. & CASLEO+REOSC \\
\hline 2454852.0 & 12.005 & $-0.05 \pm 0.01$ & N.M. & CASLEO+REOSC \\
\hline 2454853.0 & 12.005 & $-0.02 \pm 0.01$ & N.M. & CASLEO+REOSC \\
\hline 2454853.7 & 12.006 & $-0.13 \pm 0.11$ & N.M. & SOAR+Goodman \\
\hline 2454854.0 & 12.006 & $-0.12 \pm 0.11$ & N.M. & CASLEO+REOSC \\
\hline 2454854.8 & 12.006 & $+0.01 \pm 0.20$ & N.M. & LCO+MIKE \\
\hline 2454855.0 & 12.006 & $-0.07 \pm 0.11$ & N.M. & CASLEO+REOSC \\
\hline 2454859.8 & 12.009 & $-0.42 \pm 0.11$ & -90 & SOAR+Goodman \\
\hline 2454871.5 & 12.014 & $-0.76 \pm 0.20$ & -50 & $\mathrm{LCO}+\mathrm{MIKE}$ \\
\hline 2454871.7 & 12.014 & $-0.70 \pm 0.11$ & -209 & SOAR+Goodman \\
\hline 2454882.6 & 12.020 & $-1.18 \pm 0.12$ & -15 & OPD+Coudé \\
\hline 2454883.7 & 12.021 & $-1.13 \pm 0.20$ & 0. & $\mathrm{LCO}+\mathrm{MIKE}$ \\
\hline 2454888.5 & 12.023 & $-1.03 \pm 0.12$ & +6 & SOAR+Goodman \\
\hline 2454898.0 & 12.027 & $-0.11 \pm 0.08$ & 0 & CASLEO+EBASIM \\
\hline 2454899.5 & 12.028 & $-0.24 \pm 0.11$ & +3 & SOAR+Goodman \\
\hline 2454905.0 & 12.031 & $-0.40 \pm 0.33$ & 0 & CASLEO+REOSC \\
\hline 2454906.0 & 12.031 & $-0.20 \pm 0.07$ & 0 & CASLEO+REOSC \\
\hline 2454907.0 & 12.032 & $-0.18 \pm 0.32$ & 0 & CASLEO+REOSC \\
\hline 2454925.5 & 12.041 & $-0.10 \pm 0.11$ & +7 & SOAR+Goodman \\
\hline 2454940.5 & 12.048 & $-0.32 \pm 0.11$ & +0 & OPD+Coudé \\
\hline 2454942.5 & 12.049 & $-0.07 \pm 0.11$ & -9 & SOAR+Goodman \\
\hline 2454953.5 & 12.055 & $-0.06 \pm 0.11$ & -21 & SOAR+Goodman \\
\hline 2454953.6 & 12.055 & $-0.00 \pm 0.11$ & +13 & ESO/La Silla+FEROS \\
\hline 2454955.6 & 12.056 & $-0.01 \pm 0.11$ & +13 & ESO/La Silla+FEROS \\
\hline 2454967.5 & 12.062 & $0.02 \pm 0.11$ & +14 & SÓAR+Goodman \\
\hline 2455368.4 & 12.260 & $-0.05 \pm 0.14$ & -100 & OPD+Coudé \\
\hline 2455754.8 & 12.451 & $-0.10 \pm 0.10$ & +30 & OPD+Coudé \\
\hline
\end{tabular}

(a) Velocity of the highest point of the fitting of the He II $\lambda 4686$ line profile. In this table, the flag N.M. (not measurable) indicates when the line is absent (or very weak) and when the peak velocity is impossible to measure. 